\title{
NMDAR in bladder smooth muscle is not a pharmacotherapy target for overactive bladder in mice
}

\author{
Xiang Xie ${ }^{1}$, Chuang Luo ${ }^{1}$, JiaYu Liang ${ }^{1}$, Run Huang ${ }^{1}$, jia li Yang ${ }^{1}$, Linlong Li ${ }^{1}$, YangYang Li ${ }^{1}$, Hongming Xing ${ }^{1}$, \\ Huan Chen ${ }^{\text {Corresp. } 1,2}$ \\ 1 Public Center of Experimental Technology and The School of Basic Medical Sciences, Southwest Medical University, Luzhou, Sichuan, China \\ 2 Department of Medicine, Beth Israel Deaconess Medical Center and Harvard Medical School, Boston, Massachusetts, United States \\ Corresponding Author: Huan Chen \\ Email address: huanchen@swmu.edu.cn
}

Overactive bladder $(O A B)$ is a common condition that affects a significant patient population. The N-methyl-D-aspartate receptor (NMDAR) has a role in developing bladder overactivity, pharmacological inhibition of which inhibits bladder overactivity. The common pathogenesis of OAB involves bladder smooth muscle (BSM) overactivity. In this study, a smooth muscle-specific NMDAR knockout (SMNRKO) mouse model was generated. The bladders from SMNRKO mice displayed normal size and weight with an intact bladder wall and well-arranged BSM bundles. Besides, SMNRKO mice had normal voiding patterns and urodynamics and BSM contractility, indicating that NMDAR in BSM was not essential for normal physiological bladder morphology and function. Unexpectedly, cyclophosphamide (CYP)-treated SMNRKO and wild-type (WT) mice had similar pathological changes in the bladder. Furthermore, SMNRKO mice displayed similar altered voiding patterns and urodynamic abnormalities and impaired BSM contractility compared with WT mice after CYP treatment. MK801 partially reversed the pathological bladder morphology and improved bladder dysfunction induced by CYP, but did not cause apparent differences between WT mice and SMNRKO mice, suggesting that NMDAR in BSM was not involved in pathological bladder morphology and function. Moreover, the direct instillation of NMDAR agonists or antagonists into the CYP-induced OAB did not affect bladder urodynamic function, indicating that NMDAR in BSM was not the pharmacotherapy target of MK801 for CYP-induced cystitis. The findings indicated that NMDAR in BSM was not essential for normal physiological or pathological bladder morphology and function, and MK801 improving pathological bladder function was not mediated by an action on NMDAR in BSM. 
1 NMDAR in bladder smooth muscle is not a pharmacotherapy target for overactive bladder 2 in mice

3

4 Xiang Xie ${ }^{1}$, Chuang Luo ${ }^{1}$, Jiayu Liang ${ }^{1}$, Run Huang ${ }^{1}$, Jiali Yang ${ }^{1}$, Linlong Li ${ }^{1}$, Yangyang Li $^{1}$, 5 Hongming Xing ${ }^{1}$, Huan Chen ${ }^{1,2 *}$

7 'Public Center of Experimental Technology and The School of Basic Medical Sciences, Southwest 8 Medical University, Luzhou, Sichuan Province 646000, China. Electronic address: 9 huanchen@swmu.edu.cn.

$10{ }^{2}$ Department of Medicine, Beth Israel Deaconess Medical Center and Harvard Medical School, 11 Boston, Massachuesetts

13 Corresponding Author:

14 Huan Chen

15 The School of Basic Medical Sciences,

16 Southwest Medical University,

17 Luzhou, Sichuan Province 646000, China.

18 Electronic address: huanchen@swmu.edu.cn. 


\section{Abstract}

26 Overactive bladder $(\mathrm{OAB})$ is a common condition that affects a significant patient population. The 27 N-methyl-D-aspartate receptor (NMDAR) has a role in developing bladder overactivity, 28 pharmacological inhibition of which inhibits bladder overactivity. The common pathogenesis of 29 OAB involves bladder smooth muscle (BSM) overactivity. In this study, a smooth muscle-specific NMDAR knockout ( $S M N R K O)$ mouse model was generated. The bladders from SMNRKO mice displayed normal size and weight with an intact bladder wall and well-arranged BSM bundles. Besides, SMNRKO mice had normal voiding patterns and urodynamics and BSM contractility, indicating that NMDAR in BSM was not essential for normal physiological bladder morphology and function. Unexpectedly, cyclophosphamide (CYP)-treated SMNRKO and wild-type (WT) mice had similar pathological changes in the bladder. Furthermore, SMNRKO mice displayed similar altered voiding patterns and urodynamic abnormalities and impaired BSM contractility compared with WT mice after CYP treatment. MK801 partially reversed the pathological bladder morphology and improved bladder dysfunction induced by CYP, but did not cause apparent differences between WT mice and SMNRKO mice, suggesting that NMDAR in BSM was not involved in pathological bladder morphology and function. Moreover, the direct instillation of NMDAR agonists or antagonists into the CYP-induced OAB did not affect bladder urodynamic function, indicating that NMDAR in BSM was not the pharmacotherapy target of MK801 for CYPinduced cystitis. The findings indicated that NMDAR in BSM was not essential for normal physiological or pathological bladder morphology and function, and MK801 improving pathological bladder function was not mediated by an action on NMDAR in BSM.

Keywords: Bladder smooth muscle, cyclophosphamide, cystometry, MK801, N-methyl-Daspartate receptor, overactive bladder, voiding spot assay

Introduction

The International Continence Society defines overactive bladder (OAB) as a complex of symptoms 
53 nocturia (Abrams et al., 2002). OAB is a common condition in the US, with a reported prevalence 54 of $16 \%$ for men and $16.9 \%$ for women (Stewart et al., 2003). In Asia, the prevalence of OAB is 55 $19.5 \%$ in men and $22.1 \%$ in women (Chuang et al., 2019). The symptoms associated with OAB can significantly impair the quality of life and affect the social, psychological, occupational, domestic, physical, and sexual aspects of those who suffer from it. Current pharmacotherapies for 58 $\mathrm{OAB}$ include antimuscarinic and $\beta 3$-adrenoceptor agonist medications. However, these medications have either limited efficacy or significant side effects and hence many patients with OAB reject these drugs (Andersson and Wein, 2004; Chapple et al., 2008). The N-methyl-Daspartate receptor (NMDAR) is involved in developing bladder overactivity (Ishida et al., 2003; Tanaka et al., 2003; Liu et al., 2015). The pharmacological blockage of NMDAR using the noncompetitive antagonist MK801 inhibits bladder overactivity and improves bladder function (Ishida et al., 2003; Tanaka et al., 2003; Liu et al., 2015). Improvements in the understanding of NMDAR in bladder function may facilitate the discovery of new pharmacotherapies for OAB.

NMDAR is overexpressed and overactivated within irritable bowel syndrome and $\mathrm{OAB}$ and mediates visceral hyperactivity (Gaudreau et al., 2004; Yokoyama, 2010). The common pathogenesis of irritable bowel syndrome and OAB involves smooth muscle overactivity (Cukier et al., 1997; Kanazawa et al., 2014). The role of intracellular calcium is of particular importance in smooth muscle overactivity, as it is the main determinant of smooth muscle contractile activity. NMDAR has an ionotropic property that regulates calcium influx and calcium-dependent physiological effects. NMDAR in airway smooth muscle (ASM) mediates airway contractile responses and airway hyperreactivity (Anaparti et al., 2015), overactivation of which promotes vascular remolding and pulmonary arterial hypertension (PAH) (Dumas et al., 2018). However, the NDMAR knockout in smooth muscle cells or the pharmacological inhibition of NMDAR has beneficial effects on cardiac and vascular remodeling and improves PAH (Dumas et al., 2018). However, the role of NMDAR in bladder smooth muscle (BSM), thereby regulating bladder function, has not been investigated.

A smooth muscle-specific NMDAR knockout (SMNRKO) mouse model was generated in this study by mating Grin $1^{f l f l}$ (Grin1 encoding GluN1) mice with Sm22 $\alpha$-cre mice to determine 81 the role and effect of NMDAR in BSM function. As all of the major NMDAR heterotetramers are 82 thought to contain two GluN1, this strategy theoretically created an NMDAR-null in BSM. The 
83 bladder of this mouse model was then investigated by the combination of void spot assay (VSA), 84 cystometrogram (CMG), myography, and morphological approaches. The study further examined 85 the role of NMDAR in BSM under pathological conditions. Cyclophosphamide (CYP) was used 86 to induce cystitis in wild-type mice and SMNRKO mice, and comparative studies between these 87 two mouse models were performed. Moreover, the study investigated the effects of NMDAR 88 agonists and antagonists on CYP-induced cystitis through action on NMDAR in the bladder. The study provided evidence that NMDAR in BSM was not essential for normal physiological and pathological bladder morphology and function, and MK801 improving pathological bladder function was not mediated by the inhibition of NMDAR in BSM.

\section{Materials and Methods}

\section{Animals and reagents}

95

Smooth muscle NMDAR homozygous knockout mice were generated by crossing mice carrying Cre recombinase under the control of the smooth muscle transgelin promoter (SM22 $\alpha$-creKI, Stock 006878; Jackson Laboratory) with Grin $1^{f l f l}$ mice (Stock 005246; Jackson Laboratory) and then interbreeding the offspring. SM22 $\alpha$-creKI mice and Grin $1^{f l f l}$ mice were purchased from Jackson Laborarory (Bar Harbor, ME, USA). Wild-type (WT) mice were purchased form SiPeiFu Co., Ltd (Beijing, China). All mice used in this study were in the C57BL/6J background and were aged 1012 weeks. The mice were housed in standard polycarbonate cages and 5 mice per cages. The mice house environment was maintained on a 12:12-h light-dark cycle at $25{ }^{\circ} \mathrm{C}$ with humidity between $40 \%-70 \%$. The mice in cages had free access to standard laboratory food and water. Criteria for euthanizing animals before the end of the study were as follows. Under normal physiological conditions, any animals showing dying or sickness will be euthanized. Animal receiving intraperitoneal dosing will be monitored, animals showing signs of mis-dosing or dying will be euthanized. Euthanasia was performed by introduction of $100 \%$ carbon dioxide into a beddingfree cage initially containing room air with the lid closed at a rate sufficient to induce rapid anesthesia, with death occurring within 2.5 minutes. All animal care and experimental procedures were performed in adherence to the National Institutes of Health Guidelines for the Care and Use Committee of the Southwest Medical University (20180307002). Unless otherwise specified, all chemicals were obtained from Sigma (St. Louis, MO, USA) and were of reagent grade or better. 


\section{Experiment design}

115 To evaluate the voiding function of SMNRKO mice under normal physiological conditions, female

116 WT mice $(n=16)$, female SMNRKO mice $(n=22)$, male WT mice $(n=13)$, and male SMNRKO

117 mice $(\mathrm{n}=12)$ were subjected to voiding spot assay (VSA) on filter paper. Female WT mice $(\mathrm{n}=5)$

118 and female SMNRKO mice $(n=5)$ were then assessed for urodynamic function by CMG study. To

119 further determine the BSM contractility of SMNRKO mice, myography was performed on isolated

120 strips from WT mice and SMNRKO mice. Isolated strips from female WT mice (n=6), isolated

121 strips from female SMNRKO mice $(n=6)$, isolated strips from male WT mice $(n=8)$, and isolated

122 strips from male SMNRKO mice $(\mathrm{n}=8)$ were subjected to electrical field stimulation (EFS), $\mathrm{KCl}$

123 stimulation, $\alpha, \beta$-meATP stimulation, and carbachol stimulation, respectively. Additional mice

124 were also euthanized for Masson's trichrome staining ( $\mathrm{n}=3$ for female WT mice, $\mathrm{n}=3$ for female

125 SMNRKO mice, $\mathrm{n}=3$ for male $\mathrm{WT}$ mice and $\mathrm{n}=3$ for male SMNRKO mice) and

126 immunofluorescence staining $(n=3$ for female WT mice, $n=3$ for female SMNRKO mice, $n=3$ for

127 male WT mice and $\mathrm{n}=3$ for male SMNRKO mice).

128 To study the role and effects of NMDAR in BSM under pathological conditions, cystitis was 129 induced in female WT and SMNRKO mice by intraperitoneal CYP injection at a single dose of $130300 \mathrm{mg} \mathrm{kg}^{-1}$ body weight. Immediately after CYP injection, the MK801 treated cystitis group was 131 administered with MK801 at a dose of $3 \mathrm{mg} \mathrm{kg}^{-1}$ body weight. The mice survived for $48 \mathrm{~h}$ and 132 were subsequently subjected to void spot assay (VSA), cystometrogram (CMG), myography, and 133 Masson's trichrome staining studies. Specifically, Female WT mice treated with CYP (WT-CYP, $134 \mathrm{n}=10$ ), female SMNRKO mice treated with CYP (SMNRKO-CYP, $\mathrm{n}=10$ ), female WT mice 135 treated with CYP and MK801 (WT-CYP-MK801, n=12), and female SMNRKO mice treated with 136 CYP and MK801 (SMNRKO-CYP-MK801, n=11) were subjected VSA on filter paper. WT-CYP 137 mice $(n=5)$, SMNRKO-CYP mice ( $n=5)$, WT-CYP-MK801 mice $(n=5)$, and SMNRKO-CYP138 MK801 mice $(n=5)$ were then assessed for urodynamic function by CMG. Isolated strips from 139 WT-CYP mice $(n=9)$, isolated strips from SMNRKO-CYP mice $(n=9)$, isolated strips from WT140 CYP-MK801 mice $(n=9)$, and isolated strips from SMNRKO-CYP-MK801 mice (n=9) were 141 subjected to electrical field stimulation (EFS), $\mathrm{KCl}$ stimulation, $\alpha, \beta$-meATP stimulation, and 142 carbachol stimulation, respectively. The remaining mice were euthanized for Masson's trichrome 
143 staining ( $\mathrm{n}=3$ for WT-CYP mice, $\mathrm{n}=3$ for SMNRKO-CYP mice, $\mathrm{n}=3$ for WT-CYP-MK801 mice, 144 and $\mathrm{n}=3$ for SMNRKO-CYP-MK801 mice).

145 To study whether the direct instillation of NMDAR agonists or antagonists into the bladder 146 had effects on bladder urodynamic function, CMG studies were performed on female WT mice 147 pretreated with CYP during the bladder infused with NMDAR agonists or antagonists, including $148100 \mu$ M NMDA ( $=4), 100 \mu$ M RS-tetrazol-5-yl glycine ( $\mathrm{n}=4), 100 \mu$ M D-AP5 $(\mathrm{n}=4), 100$ $149 \mu \mathrm{M}$ CGS $19755(\mathrm{n}=4)$, and $100 \mu \mathrm{M}$ MK $801(\mathrm{n}=6)$.

\section{Void spot assay (VSA)}

152 Individual mice were gently placed in a standard mouse cage with Blicks Cosmos Blotting Paper 153 (Catalog no. 10422-1005) placed at the bottom for $4 \mathrm{~h}$, during which time water was withheld and 154 standard dry mouse chow was available. The mice were then returned to their home cages, and the 155 filter paper was recovered. The same individual mice were then used to repeat the experiment the 156 next day. Filters were imaged under ultraviolet light at $365 \mathrm{~nm}$ in a UVP Chromato-Vue C-75 157 system (UVP, CA, USA) with an onboard Canon digital single-lens reflex camera (EOS Rebel T3, 15812 megapixels). Overlapping voiding spots were visually examined and manually separated by 159 outlining and copying, and then pasted on to a nearby empty space using ImageJ software 160 (http://fiji.sc/wiki/index.php/Fiji). The images were analyzed using UrineQuant software 161 developed in collaboration with the Harvard Imaging and Data Core. The result table containing 162 the area of each individual voiding spot and the total number of spots was imported into Excel for 163 statistical analysis. A volume:area standard curve showed that a $1-\mathrm{mm}^{2}$ voiding spot represents $1640.283 \mu \mathrm{L}$ of urine. Voiding spots with an area $\geq 80 \mathrm{~mm}^{2}$ were considered primary voiding spots 165 (PVS) based on voiding spot patterns from hundreds of mice (Chen et al., 2017; Rajandram et al., 166 2016).

\section{Cystometrogram (CMG)}

169 CMG was performed with PBS infusion $\left(25 \mu \mathrm{L} \mathrm{min}^{-1}\right)$ as previously described (Hao et al., 2019).

170 The mice were anesthetized by subcutaneously injecting urethane $\left(1.4 \mathrm{~g} \mathrm{~kg}^{-1}\right.$ from $250 \mathrm{mg} \mathrm{mL}-1$ 
171 solution in PBS) 30-60 min before the surgery. At the time of surgery, the mice were further 172 anesthetized with continuous-flow isoflurane (3\% induction, 1.0\% maintenance). Once the pedal 173 reflex was absent, a 1-cm midline abdominal incision was performed. Flame-flanged polyethylene-

17450 tubing sheathing a $25 \mathrm{G} \times 1.5$ in. needle was implanted through the dome of the bladder and 175 then sutured in place (8-0 silk purse-string sutures). The incision site was sutured around the tubing 176 using sterile 5-0 silk sutures, and the mice were then placed into a Bollman mouse restrainer for 17730 - to 60-min stabilization. The catheter was connected to a pressure transducer (and syringe pump 178 by the side arm) coupled to data-acquisition devices (WPI Transbridge and AD Instruments 179 PowerLab 4/35) and a computerized recording system (AD Instruments LabChart software). 180 Bladder filling then commenced, following which voiding occurred naturally through the urethra. 181 Repeated voiding cycles were assessed for the change in the voiding interval (time between peak 182 pressures), basal pressure (minimum pressure after voiding), threshold pressure (pressure 183 immediately before the onset of voiding contraction), peak pressure (maximum voiding pressure

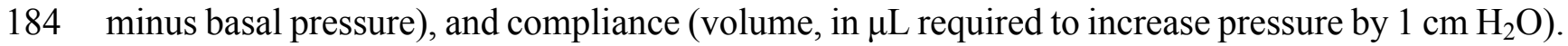

\section{Myograph}

187 Bladders were pinned on a small Sylgard block, and the muscle was dissected free of the mucosal tissue. BSM strips were then cut longitudinally ( $2 \mathrm{~mm}$ wide and $7 \mathrm{~mm} \mathrm{long}$ ). Muscle strips were mounted in an SI-MB4 tissue bath system (World Precision Instruments, FL, USA). Force sensors were connected to a TBM 4M transbridge (World Precision Instruments), and the signal was amplified by PowerLab (AD Instruments, CO, USA) and monitored through Chart software (AD Instruments). BSM strips were gently prestretched to optimize contraction force and then preequilibrated for at least $1 \mathrm{~h}$. All experiments were conducted at $37{ }^{\circ} \mathrm{C}$ in physiological saline solution (in mM: $120 \mathrm{NaCl}, 5.9 \mathrm{KCl}, 1.2 \mathrm{MgCl}_{2}, 15.5 \mathrm{NaHCO}_{3}, 1.2 \mathrm{NaH}_{2} \mathrm{PO}_{4}, 11.5$ glucose, and $2.5 \mathrm{mM} \mathrm{CaCl}_{2}$ ) with continuous bubbling of $95 \% \mathrm{O}_{2} / 5 \% \mathrm{CO}_{2}$. Contraction force was sampled at

196 2000/s using Chart software. BSM tissue was treated with agonists or antagonists and/or subjected 197 to electrical field stimulation (EFS). BSM strip EFS was carried out using a Grass S48 field 198 stimulator (Grass Technologies, RI, USA) using previously described standard protocols ( 199 Rajandram et al., 2016; Chen et al., 2020). 


\section{Histological and immunofluorescence staining}

202 For histopathological evaluation, the bladders were removed under anesthesia, fixed in 4\%

203 formaldehyde, embedded in paraffin blocks, and sectioned. The sections were stained with 204 Masson's trichrome staining. For immunofluorescence staining, excised bladders were fixed in $2054 \%(\mathrm{w} / \mathrm{v})$ paraformaldehyde for $2 \mathrm{~h}$ at room temperature. The fixed tissue was cryoprotected, 206 frozen, sectioned, and incubated with a purified rat anti-mouse $\beta 1$ integrin antibody (1:100, 207 \#550531, BD Bioscience) overnight at $4{ }^{\circ} \mathrm{C}$. The sections were then incubated with an Alexa Fluor 208 488-conjugated secondary antibody (diluted 1:100), and nuclei were stained with DAPI. Imaging 209 was performed on an Olympus BX60 fluorescence microscope with a 40×/0.75 objective. Images 210 (512 and 512 pixels) were saved as TIFF files and imported into an Adobe Illustrator CS3. In the

211 present study, each bladder was sectioned to obtain two slides with approximately four sections of 212 tissue on each slide. Each tissue section was examined under a microscope to ensure the 213 consistency of the staining result, and images were taken and quantitated.

215 Statistical analyses

216 All data were presented as boxes and whiskers. The centerline was the median of the data set, the 217 box represented $75 \%$ of the data, and the bars indicated whiskers from minimum to maximum. 218 Data were analyzed by the Student $t$-test between the two groups using GraphPad Prism 8 software, 219 and a $\mathrm{P}$ value $<0.05$ was considered significant.

\section{Results}

\section{SMNRKO mice had normal bladder morphology}

223 SM22 $\alpha$-Cre mice were crossed with Grin $1^{f l f l}$ mice to obtain smooth muscle-specific knockout 224 protein. The deletion of the Grinl gene was verified by genomic DNA genotyping and mRNA 225 expression analysis (data are shown in upload Raw data). Bladders from female and male 226 SMNRKO mice both displayed normal size and weight (Fig. 1A, C). Since SMNRKO mice of both 
227 sexes had no obvious changes in body weight (Fig. 1B), the ratios of bladder weight (mg) to body 228 weight (g) revealed no difference between SMNRKO mice and wild-type mice (Fig. 1D). Masson's 229 trichrome-stained bladder sections from female and male SMNRKO mice were examined to 230 further investigate whether the histology changed in the bladder of SMNRKO mice. As shown in

231 Fig. 1E-N, the histology of the bladder from both sexes revealed no observed differences between 232 SMNRKO mice and wild-type (WT) mice. The bladder wall of WT mice and SMNRKO mice had 233 similar thickness and integrity; no evidence indicated derangement or separation (Fig. 1E, F, K, 234 and L). The smooth muscle layer showed compact well-arranged smooth muscle bundles with 235 collagen fibers in between (Fig. 1G, H, M, and N). The thickness of the smooth muscle layer from 236 both sexes of SMNRKO mice revealed no differences compared with that of WT mice. 237 Furthermore, $\beta$-integrin immune-stained sections revealed intact, well-distributed smooth muscle 238 cells with normal size (Fig. 1I, J, O, and P). In summary, these data suggested that NMDAR in 239 BSM did not contribute to bladder morphology in mice.

\section{SMNRKO mice exhibited normal urinary function and BSM contractility}

242 VSA was performed in female and male mice to evaluate whether NMDAR in BSM was involved 243 in regulating bladder voiding function. Female $S M N R K O$ mice produced approximately 3.68 PVS 244 with an average size of $\sim 357.20 \mathrm{~mm}^{2} /$ spot in a 4 -h period, which showed no significant difference compared with female WT mice (Fig. 2A, B, E, and F). Besides, they had similar total voiding 246 volume (Fig. 2G). To show shifts in the overall pattern of voiding behavior, all the spots over 40 $247 \mathrm{~mm}^{2}$ were grouped into different sizes with their frequency distribution. Female $S M N R K O$ mice 248 and female WT mice showed a similar voiding pattern (Fig. 2H). The size-frequency chart of voiding spots showed a distinct voiding size distribution between male SMNRKO mice and WT mice (Fig. 2I). However, the VSA of male SMNRKO mice indicated the grossly same PVS number, PVS size, and total voiding volume as that of male WT mice (Fig. 2C, D, E, F, and G), suggesting that male SMNRKO mice had normal bladder voiding function. Next, CMGs were performed on female SMNRKO mice to evaluate bladder urodynamic function. Fig. 2J and K showed CMG traces in which time-dependent bladder pressure changes were recorded during continuous filling and emptying cycles in WT mice and SMNRKO mice, respectively. However, none of the analyzed cystometric parameters of $S M N R K O$ mice showed any significant differences compared with those 
257 of WT mice. These included voiding interval, basal pressure, micturition threshold pressure, peak 258 pressure, and bladder compliance (Fig. 2L-P), thus further confirming that SMNRKO mice had 259 normal bladder function. Myography was performed on isolated strips from WT mice and

260 SMNRKO mice to determine whether NMDAR in BSM affected BSM contractility. As shown in 261 Fig. 3A-F, the contractile force of BSM strips from female and male SMNRKO mice increased 262 with EFS frequency. However, the average force under each EFS frequency showed no significant 263 differences compared with WT mice. Furthermore, BSM strips from SMNRKO mice of both sexes 264 had normal contractility in response to carbachol, a,b-meATP, and $\mathrm{KCl}$ depolarization (Fig. 3G265 I). In summary, these data suggested that NMDAR in BSM did not contribute to normal 266 physiological bladder function in mice.

\section{NMDAR in BSM was not essential for pathological bladder morphology}

269

270

271

272

273

274

275

276

277

278

279

280

281

282

283

284

285

286

Pharmacological NMDAR blockage using the noncompetitive antagonist MK801 has beneficial effects on cardiac, vascular, and bladder remodeling under pathological conditions (Liu et al., 2015; Dumas et al., 2018). However, whether NMDAR deficiency in BSM had a beneficial role in bladder remodeling under pathological conditions is unclear. To answer this, the present study investigated the morphology of CYP-induced cystitis bladder and MK801-treated cystitis bladder from female WT mice and SMNRKO mice. As shown in Fig. 4A-D, the bladders from SMNRKO mice and WT mice were significantly larger and heavier after CYP treatment, indicating bladder hypertrophy. Besides, CYP treatment resulted in a markedly reduced body weight and a significant increase in the ratio of bladder weight to body weight (Fig. 4D). However, these changes revealed no differences between SMNRKO mice and WT mice (Fig. 4B-D). In CYP-treated WT mice and SMNRKO mice, the administration of MK801 reversed bladder weight and size (Fig. 4A-D), but still no differences were observed between WT mice and SMNRKO mice (Fig. 4A-D). Masson's trichrome staining was then used to investigate the bladder pathological changes. The images of stained tissue from SMNRKO mice and WT mice both displayed dramatic structural changes after CYP treatment. As shown in Fig. 4E-L, the urothelial layer appeared much thinner with the disrupted superficial inner layer (Fig. 4F and J). The lamina propria layer was hugely thickened, and the vasculature within the lamina propria was dramatically dilated (Fig. 4G and K). The muscle bundles were disorganized with obvious injury, and interstitial edema among muscle bundles was 
287 obvious with enlarged spaces (Fig. 4H and L). Importantly, the administration of MK801 partially 288 reversed these CYP-induced changes in both WT mice and SMNRKO mice. As shown in Fig. 4M289 T, the urothelial layer appeared much thicker with no obvious disruption (Fig. 4N and R). The 290 lamina propria layer was thinner, and the vasculature within the lamina propria was dilated but 291 with a few numbers (Fig. 4 O and S). The muscle bundles were well organized, and the interstitial 292 edema among muscle bundles was not apparent (Fig. 4P and T). Although the administration of 293 MK801 reversed CYP-induced pathological changes in both WT mice and SMNRKO mice, 294 bladders from both mice displayed similar histological changes without any significant 295 differences. In summary, these data suggested that NMDAR in BSM was not essential for bladder 296 morphology under pathological conditions.

\section{NMDAR in BSM was not involved in pathological bladder function and contractility}

299

300

301

302

303

304

305

306

307

308

309

310

311

312

313

314

315

316

NMDAR inhibition with MK801 is effective in improving cardiac, vascular, and bladder function under pathological conditions (Liu et al., 2015; Dumas et al., 2018). Therefore, the present study investigated the voiding function of CYP-induced cystitis bladder and MK801-treated cystitis bladder from female $S M N R K O$ mice and WT mice to examine whether NMDAR deficiency in BSM improved pathological bladder function. As shown in Fig. 5A-G, SMNRKO mice and WT mice both had compromised bladder function after CYP treatment, characterized by significantly smaller spots and increased numbers of individual void spots. However, no marked differences were observed in analyzed VSA parameters between WT mice and SMNRKO mice after CYP treatment (Fig. 5A-G). Besides, the size-frequency chart of voiding spots from both mice showed the same altered voiding pattern, which reached the top in an area of $40-80 \mathrm{~mm}^{2}$ and then decreased with the increase in the area (Fig. 5H). In CYP-treated WT mice and SMNRKO mice, all these voiding abnormalities were abrogated after MK801 treatment, as manifested by decreased numbers of individual void spots and increased void spot size (Fig. 5C-G). In addition, voiding spots of both mice after MK801 treatment showed a relatively normal voiding pattern similar to those in normal WT mice and SMNRKO mice (Fig. 5H). However, the MK801-treated SMNRKO mice still displayed no difference in voiding function compared with MK801-treated WT mice (Fig. 5C-H), indicating that NMDAR in BSM did not contribute to bladder function under pathological conditions, which was further supported by CMGs. As shown in Figure 5I-Q, CYP- 
317 treated WT mice and SMNRKO mice both exhibited similar urodynamic abnormalities with

318 decreased voiding interval, peak pressure, and compliance and increased basal pressure (Fig. 5M-

319 Q). After MK801 treatment, both mice retained a relatively normal urodynamic function with 320 similar normal cystometric parameters (Fig. 5M-Q). Further, myography was performed to 321 determine whether NMDAR deficiency in BSM affected BSM contractility under pathological 322 conditions. Fig. 6A-H shows that BSM strips from SMNRKO mice and WT mice after CYP 323 treatment had impaired contractility in response to EFS, carbachol, and $\mathrm{KCl}$ depolarization. 324 However, the impaired contractility was reversed by MK801 treatment. Consistently, no 325 significant differences were found in contractility response between SMNRKO mice and WT mice 326 after MK801 treatment (Fig. 6A-H). In summary, these data suggested that NMDAR in BSM did 327 not contribute to pathological bladder function and contractility.

Pharmacotherapies targeted on NMDAR in BSM did not improve pathological bladder function

331 Intravenous injection of MK801 is an effective way to suppress CYP-induced bladder overactivity 332 (Liu et al., 2015). Whether the direct instillation of NMDAR antagonists into the bladder had the 333 same effect was not known. Hence, CMG studies were performed during NMDAR antagonist 334 infusion into CYP-induced OAB. The CYP ruptured and caused significant damage to the 335 urothelium, thus allowing the infusion drug to penetrate into the smooth muscle layer and work on 336 BSM cells. As shown in Fig 7, up to $100 \mu$ M D-AP5, CGS 19755, and MK 801 instillation did not 337 improve bladder dysfunction. The mice after receiving NMDAR antagonist instillation displayed 338 similar urodynamic abnormalities compared with the mice before receiving instillation. Besides, 339 the direct instillation of NMDAR agonists into the bladder failed to worsen bladder function in 340 CYP-induced OAB (Fig 7). These results were consistent with a recent report that revealed the 341 NMDAR agonists, including NMDA and RS-tetrazol-5-yl glycine, at concentrations up to $100 \mu \mathrm{M}$ 342 had no effect on BSM contractile force, and NMDAR antagonists, including D-AP5, CGS19755, 343 and MK801, had little or no effect on BSM contraction (Chen et al., 2020). Furthermore, the lack 344 of NMDAR in BSM had no effects on bladder morphology and function under normal or 345 pathological conditions, and SMNRKO mice with cystitis after MK801 treatment displayed no 346 differences in bladder phenotypes compared with WT mice with cystitis after MK801 treatment 
347 (Figs 4, 5, and 6). Taken together, these data suggested that pharmacological drugs targeted on

348 NMDAR in BSM did not improve pathological bladder function.

350 Discussion

351 The role of NMDAR is mainly known through its neural activity. However, its expression and role

352 in a variety of nonneural peripheral cells and organs have also been shown. In particular, NDMAR

353 is expressed throughout the cardiovascular system and plays a role in electrical and pacemaker

354 activities (Hogan and Anderson, 2016). In the lung, ASM cells expressing NMDAR enhance

355 airway responsiveness (Cukier et al., 1997; Dumas et al., 2018; Quatredeniers et al., 2019) and

356 contribute directly to bronchiole smooth muscle contraction (Antosova and Strapkova, 2015;

357 Anaparti et al., 2015). NMDAR subunit mRNA and proteins have also been found to be expressed

358 in the lower urogenital tract and may participate in the control of organ tone for male sexual

359 activity (Gonzalez et al., 2000). Emerging evidence shows that NMDAR is involved in the

360 development of $\mathrm{OAB}$, pharmacological inhibition of which markedly reduced bladder overactivity

361 and improved bladder function (Liu et al., 2015). BSM from OAB often shows enhanced

362 spontaneous contractile activity, which has been documented in human bladder strips from

363 obstructed unstable bladders and patients with neuropathy (Sibley, 1984; German et al., 1993;

364 Steers, 2002). NMDAR previously studied in smooth muscle is associated with airway remolding

365 and contributes to airway hyperreactivity (Anaparti et al., 2015). This study investigated the roles

366 and effects of NMDAR in BSM on the regulation of bladder morphology and function under

367 normal physiological and pathological conditions. The inhibition of NMDAR by injection of

368 MK801 had positive effects on bladder remodeling and bladder function, but the deficiency of

369 NMDAR in BSM had no such effects. Furthermore, the study verified that NDMAR in BSM was

370 not the pharmacotherapy target for CYP-induced cystitis.

371 Smooth muscle-specific Grinl knockout mice were generated in the present study. Grinl 372 gene encodes GluN1, which is a subunit of NMDAR. Previous studies found that several NDMAR 373 isoforms were expressed in human and mouse bladder (Yue et al., 2014; Fagerberg et al., 2014).

374 The findings were consistent with a most recent study showing that BSM cells expressed NMDAR

375 subunits, which were verified by the single-cell sequencing method (Yu et al., 2019). NMDAR is 
376 composed of four subunits: two obligatory subunits GluN1 and two variable subunits including 377 GluN-2A/2B/2C/2D/3A/3B. Therefore, the knockout of obligatory subunit GluN1 in BSM 378 theoretically created an NMDAR-null in BSM. This strategy was previously used in the lung to 379 study the role of NMDAR in ASM (Dumas et al., 2019). The bladders from SMNRKO mice 380 showed normal size and weight, an intact bladder wall with different layers, and well-arranged 381 BSM bundles with normal size smooth muscle cells, indicating that the deficiency of NMDAR in 382 BSM under normal physiological conditions did not impact BSM cells and change bladder 383 morphology. Unexpectedly, SMNRKO mice had normal voiding patterns and urodynamic 384 function, indicating normal bladder function. BSM contractility is essential for physiological 385 bladder function (Hao et al., 2019; Chen et al., 2020). The study further found that BSM strips 386 from $S M N R K O$ mice exhibited normal contractility, which was consistent with previous findings 387 that the NMDAR agonists and antagonists had little or no effect on BSM contraction (Chen et al., 388 2020), indicating that NMDAR in BSM did not regulate BSM contractility, which explained why 389 the loss of NMDAR in BSM had normal bladder function. Consistently, Dumas also reported that 390 no differences were found in vascular remodeling and pulmonary function under normoxic 391 conditions between SMNRKO mice and WT mice (Dumas et al., 2019). NMDAR subunits might 392 not be activated or form fully active receptors in BSM under normal physiological conditions, 393 deficiency of which had no effects on bladder morphology and function.

394 NMDAR activation is commonly found to be associated with pathological conditions. For 395 example, NMDAR activation was found to mediate visceral hyperactivity in irritable bowel 396 syndrome and OAB (Gaudreau and Plourde, 2004; Yokoyama, 2010). In the cerebral or aortic 397 endothelium, NMDAR activation contributed to endothelial barrier disruption, inflammatory cell 398 infiltration, oxidative stress, and proliferation (Sharp et al., 2003; Chen et al., 2005; Andras et al., 399 2007; Reijerkerk et al., 2010). In aortic and ASM cells, NMDAR activation stimulated cell 400 proliferation, promoted cardiac and vascular remodeling, and induced PAH(Qureshi et al., 2005; 401 Doronzo et al., 2010; Anaparti et al., 2015; Dumas et al., 2018). In contrast, the targeted knockout 402 of NDMAR in ASM had beneficial effects on cardiac and vascular remolding and improved PAH 403 (Dumas et al., 2019). The aforementioned studies suggested that NDMAR activation played roles 404 in pathological conditions, deficiency of which had beneficial effects. However, in the present 405 study, both SMNRKO mice and WT mice showed marked histological changes within the bladder 406 after CYP treatment. Unexpectedly, NMDAR deficiency in BSM did not improve pathological 
407 bladder remolding. Furthermore, CYP-treated SMNRKO mice displayed similar altered voiding 408 patterns and urodynamic abnormalities and impaired BSM contractility as CYP-treated WT mice, 409 suggesting that NMDAR deficiency in BSM did not improve pathological bladder function either.

410 Liu et al. previously demonstrated that NDMAR activation was primarily present in the central 411 nervous system but not in the bladder during CYP-induced cystitis (Liu et al., 2015), indicating 412 that CYP-induced cystitis did not stimulate NMDAR activation in the bladder. Thus, NMDAR in 413 BSM was not involved in CYP-induced pathological bladder morphology and function.

414 The pharmacological inhibition of NMDAR by the intravenous injection of MK801 has been 415 previously reported to inhibit bladder overactivity caused by cerebral infarction (Yotsuyanagi et 416 al 2005) and attenuate hyperreflexia in the micturition reflex induced by crystalluria, partial 417 bladder outlet obstuction (BOO), and nerve injury (Ishida et al., 2003; Tanaka et al., 2003; 418 Yokoyama et al., 2004; Kontani and Ueda, 2005). In addition, a most recent study reported that 419 the injection of MK801 reduced bladder hypertrophy and suppressed bladder overactivity induced 420 by CYP (Liu et al., 2015), suggesting that MK801 was effective for OAB treatment in mice. 421 Consistently, in the present study, the injection of MK801 reversed pathological bladder histology 422 and rescued bladder dysfunction in CYP-treated WT mice and SMNRKO mice. However, WT 423 mice still displayed a similar bladder morphology, voiding pattern, urodynamics, and BSM 424 contractility as $S M N R K O$ mice after MK801 treatment, suggesting that NMDAR in BSM was not 425 the treatment target of MK 801 for CYP-induced cystitis, which was further supported by the 426 results of CMGs during the direct instillation of NMDAR agonists or antagonists into the CYP427 induced cystitis bladder. The direct instillation of NDMAR antagonists, including MK801, D-AP5, 428 and CGS 19755, into the bladder did not correct pathological bladder urodynamics. The instillation 429 of NMDAR agonists, including NMDA and RS-tetrazol-5-yl glycine did not worse the bladder 430 urodynamics. These results were consistent with the findings of previous myography studies 431 revealing that NMDAR agonists NDMA and RS-tetrazol-5-yl glycine had no effects on BSM 432 contractile force, and NMDAR antagonists MK801, D-AP5, and CGS 19755 had little or no effects 433 on BSM contraction (Chen et al., 2020). Besides, the deficiency of NMDAR in BSM did not 434 contribute to bladder morphology and function under normal physiological and pathological 435 conditions. Therefore, it was believed that the intravenous injection of MK801 improving 436 pathological bladder morphology and function was not mediated by the inhibition of NMDAR in 437 BSM. Yoshiyama et al. suggested that the inhibition of micturition reflex by MK801 was mediated 
438 by an action on the lumbosacral spinal cord (Yoshiyama et al., 1993). Liu et al. reported that the 439 injection of D-AP5, which could not cross the blood-brain barrier and block central NMDAR 440 activity, failed to reduce the pathological bladder morphology and function during CYP-induced 441 cystitis (Liu et al., 2015). However, the injection of MK801, which crossed the blood-brain barrier 442 and inhibited central NMDAR activity, could reduce bladder hypertrophy and improve bladder 443 function in CYP-induced cystitis. Furthermore, cystitis-induced NMDAR activity was found to be 444 extremely low in the bladder compared with the spinal cord (Liu et al., 2015). Thus, NMDAR in 445 the central nervous system was a pharmacotherapy target of MK801 for CYP-induced cystitis 446 treatment, but not NMDAR in BSM.

447 The present study showed that NMDAR in BSM was not essential for normal physiological 448 and pathological bladder morphology or function, and MK801-mediated improvement in 449 pathological bladder function was not associated with NMDAR in BSM.

450

\section{Acknowledgments}

452

\section{Conflict of Interest}

454 The author(s) declare(s) that there are no conflicts of interest.

455

\section{Author Contribution}

457 Conceptualization: H.C., X.X; Methodology: H.C., X.X; Formal analysis: H.C., X.X., R.H., 458 J.Y.L., C. L., J.L.Y., L.L.L., Y.Y.L., H.M.X; Investigation: H.C., X.X., R.H., J.Y.L., C. L., J.L.Y., 459 L.L.L., Y.Y.L., H.M.X; Writing - original draft: H.C., X.X; Writing - review \& editing: H.C., X.X; 460 Supervision: H.C; Project administration: H.C; Funding acquisition: H.C.

461

462 Funding

463 This work was supported by grants from the National Natural Science Foundation of China 464 (81800670), Luzhou City Bureau of Science and Technology (2020LZXNYDZ05), National 465 Undergradute Innovation and Enterpreneurship Training program (S201910703072, 466 S202010632005, 2020360).

467

\section{References}


469

470

471

472

473

474

475

476

477

478

479

480

481

482

483

484

485

486

487

488

489

490

491

492

493

494

495

Abrams, P., Cardozo, L., Fall, M., Griffiths, D., Rosier, P., Ulmsten, U., Van Kerrebroeck, P., Victor, A., and Wein, A. (2003). Standardisation Sub-Committee of the International Continence Society. The standardisation of terminology in lower urinary tract function: report from the standardisation sub-committee of the International Continence Society. Urology. 61(1):37-49.

Anaparti, V., Ilarraza, R., Orihara, K., Stelmack, G. L., Ojo, O. O., Mahood, T. H., Unruh, H., Halayko, A. J., and Moqbel, R. (2015). NMDA receptors mediate contractile responses in human airway smooth muscle cells. Am J Physiol Lung Cell Mol Physiol. 308(12):L125364.

Andersson, K. E., and Wein, A. J. (2004). Pharmacology of the lower urinary tract: basis for current and future treatments of urinary incontinence. Pharmacol Rev. 56(4):581-631.

András, I. E., Deli, M. A., Veszelka, S., Hayashi, K., Hennig, B., and Toborek, M. (2007). The NMDA and AMPA/KA receptors are involved in glutamate-induced alterations of occludin expression and phosphorylation in brain endothelial cells. J Cereb Blood Flow Metab. 27(8):1431-43.

Antošová, M., and Strapková, A. (2013). Study of the interaction of glutamatergic and nitrergic signalling in conditions of the experimental airways hyperreactivity. Pharmacol Rep. 65(3):650-7.

Chapple, C.R, Khullar, V., Gabriel, Z., Muston, D., Bitoun, C. E., and Weinstein, D. The effects of antimuscarinic treatments in overactive bladder: an update of a systematic review and meta-analysis. Eur Urol. 54(3):543-62.

Chen. H., Fitzgerald. R., Brown. A. T., Qureshi. I., Breckenridge. J., Kazi. R., Wang. Y., Wu. Y., Zhang. X., Mukunyadzi. P., et al. (2005). Identification of a homocysteine receptor in the peripheral endothelium and its role in proliferation. J Vasc Surg. 41(5):853-60.

Chen. H., Zhang. L., Hill, W.G., and Yu, W. (2017). Evaluating the voiding spot assay in mice: a simple method with complex environmental interactions. Am J Physiol Renal Physiol. 313(6):F1274-F1280. 
496

497

498

499

500

501

502

503

504

505

506

507

508

509

510

511

512

513

514

515

516

517

518

519

520

521

Chen, H., Vandorpe, D. H., Xie, X., Alper, S. L., Zeidel, M. L., and Yu, W. (2020). Disruption of Cav1.2-mediated signaling is a pathway for ketamine-induced pathology. Nat Commun. 11(1):4328.

Chuang, Y. C., Liu, S. P., Lee, K. S., Liao, L., Wang, J., Yoo, T. K, Chu, R., and Sumarsono, B. (2019). Prevalence of overactive bladder in China, Taiwan and South Korea: Results from a cross-sectional, population-based study. Low Urin Tract Symptoms. 11(1):48-55.

Cukier, J. M., Cortina-Borja, M., and Brading, A. F. (1997). A case-control study to examine any association between idiopathic detrusor instability and gastrointestinal tract disorder, and between irritable bowel syndrome and urinary tract disorder. Br J Urol. 79(6):865-78.

Doronzo, G., Russo, I., Del Mese, P., Viretto, M., Mattiello, L., Trovati, M., and Anfossi, G. (2010). Role of NMDA receptor in homocysteine-induced activation of mitogen-activated protein kinase and phosphatidyl inositol 3-kinase pathways in cultured human vascular smooth muscle cells. Thromb Res. 125(2):e23-32.

Dumas, S. J., Bru-Mercier, G., Courboulin, A., Quatredeniers, M., Rücker-Martin, C., Antigny, F., Nakhleh, M. K., Ranchoux, B., Gouadon, E., Vinhas, M. C. et al. (2018). NMDA-Type Glutamate Receptor Activation Promotes Vascular Remodeling and Pulmonary Arterial Hypertension. Circulation. 137(22):2371-2389.

Fagerberg, L., Hallström, B. M., Oksvold, P., Kampf, C., Djureinovic, D., Odeberg, J., Habuka, M., Tahmasebpoor, S., Danielsson, A., Edlund, K. et al. (2014). Analysis of the human tissue-specific expression by genome-wide integration of transcriptomics and antibodybased proteomics. Mol Cell Proteomics. 13(2):397-406.

Gaudreau, G. A., and Plourde, V. (2004). Involvement of N-methyl-d-aspartate (NMDA) receptors in a rat model of visceral hypersensitivity. Behav Brain Res. 150(1-2):185-9.

German, K., Bedwani, J., Davies, J., Brading, AF., and Stephenson, T. P. (1995). Physiological and morphometric studies into the pathophysiology of detrusor hyperreflexia in neuropathic patients. J Urol. 153(5):1678-83. 
522 Gonzalez-Cadavid, N. F., Ryndin, I., Vernet, D., Magee, T. R., and Rajfer, J. (2000). Presence

523

524

525

526

527

528

529

530

531

532

533

534

535

536

537

538

539

540

541

542

543

544

545

546

547

548 of NMDA receptor subunits in the male lower urogenital tract. J Androl. 21(4):566-78.

Hao, Y., Wang L., Chen, H., Hill, W. G., Robson, S. C., Zeidel, M. L., and Yu W. (2019). Targetable purinergic receptors $\mathrm{P} 2 \mathrm{Y} 12$ and A2b antagonistically regulate bladder function. JCI Insight. 4(16):e122112.

Hogan-Cann, A. D., and Anderson, C. M. (2016). Physiological Roles of Non-Neuronal NMDA Receptors. Trends Pharmacol Sci. 37(9):750-767.

Ishida, F., Sato, T., Imaizumi, M., Funayama, N., Ikegami, K., Nimura, T., and Kawatani, M. (2003). MK-801 inhibits the micturition reflex in chronic bladder irritation caused by crystalluria in the rat. Auton Neurosci. 105(1):1-7.

Kanazawa, M., Palsson, O. S., van Tilburg, M. A., Gangarosa, L. M., Fukudo, S., and Whitehead, W. E. (2014). Motility response to colonic distention is increased in postinfectious irritable bowel syndrome (PI-IBS). Neurogastroenterol Motil. 26(5):696-704.

Kontani, H., and Ueda, Y. (2005). A method for producing overactive bladder in the rat and investigation of the effects of GABAergic receptor agonists and glutamatergic receptor antagonists on the cystometrogram. J Urol. 173(5):1805-11.

Liu, M., Shen S., Kendig, D. M., Mahavadi, S., Murthy, K. S., Grider, J. R., and Qiao, L. Y. (2015). Inhibition of NMDAR reduces bladder hypertrophy and improves bladder function in cyclophosphamide induced cystitis. J Urol. 193(5):1676-83.

Quatredeniers, M., Nakhleh, M. K., Dumas, S. J., Courboulin, A., Vinhas, M. C., Antigny, F., Phan, C., Guignabert, C., Bendifallah, I., Vocelle, M. et al. (2019). Functional interaction between PDGF $\beta$ and GluN2B-containing NMDA receptors in smooth muscle cell proliferation and migration in pulmonary arterial hypertension. Am J Physiol Lung Cell Mol Physiol. 316(3):L445-L455.

Qureshi, I., Chen, H., Brown, A. T., Fitzgerald, R., Zhang, X., Breckenridge, J., Kazi, R., Crocker, A. J., Stühlinger, M. C., Lin, K. et al. (2005). Homocysteine-induced vascular dysregulation is mediated by the NMDA receptor. Vasc Med. 10(3):215-23. 
549 Rajandram, R., Ong, T. A., Razack, A. H., MacIver, B., Zeidel, M., and Yu, W. (2016). Intact 550 urothelial barrier function in a mouse model of ketamine-induced voiding dysfunction. Am J 551 Physiol Renal Physiol. 310(9):F885-94.

552

553

554

555

556

557

558

559

560

561

562

563

564

565

566

567

568

569

570

571

572

573

574

575

Reijerkerk, A., Kooij G., van der Pol, S. M., Leyen, T., Lakeman, K., van Het Hof, B., Vivien, D., and de Vries, H. E. (2010). The NR1 subunit of NMDA receptor regulates monocyte transmigration through the brain endothelial cell barrier. J Neurochem. 113(2):447-53.

Sharp, C. D., Houghton, J., Elrod, J. W., Warren, A., Jackson, T. H $4^{\text {th }}$., Jawahar, A., Nanda, A., Minagar, A., and Alexander, J. S. (2005). N-methyl-D-aspartate receptor activation in human cerebral endothelium promotes intracellular oxidant stress. Am J Physiol Heart Circ Physiol. 288(4):H1893-9.

Sibley, G. N. A. (1984). The Response of the Bladder to Lower Urinary Tract Obstruction Oxford, England: Oxford University.

Steers, W. D. (2002). Pathophysiology of overactive bladder and urge urinary incontinence. Rev Urol. 4 Suppl 4(Suppl 4):S7-S18.

Stewart, W. F., Van Rooyen, J. B., Cundiff, G. W., Abrams, P., Herzog, A. R., Corey, R., Hunt, T. L., and Wein A. J. (2003). Prevalence and burden of overactive bladder in the United States. World J Urol. 20(6):327-36.

Tanaka, H., Kakizaki, H., Shibata, T., Ameda, K., and Koyanagi, T. (2003). Effects of chronic blockade of N-methyl-D-aspartate receptors by MK-801 on neuroplasticity of the micturition reflex pathway after partial urethral obstruction in the rat. J Urol. 170(4 Pt 1):1427-31.

Yokoyama, O., Mizuno, H., Komatsu, K., Akino, H., Tanase, K., and Namiki M. (2004). Role of glutamate receptors in the development and maintenance of bladder overactivity after cerebral infarction in the rat. J Urol. 171(4):1709-14.

Yokoyama, O. (2010). Pharmacological and genetic analysis of mechanisms underlying detrusor overactivity in rats. Neurourol Urodyn. 29(1):107-11.

Yoshiyama, M., Roppolo, J. R., and de Groat, W. C. (1993). Effects of MK-801 on the micturition reflex in the rat--possible sites of action. J Pharmacol Exp Ther. 265(2):844-50. 
576

577

578

579

580

581

582

583

584

585

586

587

588

589

590

591

592

593

594

595

596

597

598

599

600

601

602

603

Yotsuyanagi, S., Yokoyama, O., Komatsu, K., Kodama, K., Nagasaka, Y., and Namiki, M. (2005). Role of cyclooxygenase-2 in the development of bladder overactivity after cerebral infarction in the rat. J Urol. 174(1):365-9.

Yu, Z., Liao, J., Chen, Y., Zou, C., Zhang, H., Cheng, J., Liu, D., Li, T., Zhang, Q., Li, J. et al. (2019). Single-Cell Transcriptomic Map of the Human and Mouse Bladders. J Am Soc Nephrol. 30(11):2159-2176.

Yue, F., Cheng Y., Breschi A., Vierstra J., Wu W., Ryba T., Sandstrom R., Ma Z., Davis C., Pope BD. et al. (2014). Mouse ENCODE Consortium. A comparative encyclopedia of DNA elements in the mouse genome. Nature. 515(7527):355-64.

Figure 1. Bladders of SMNRKO mice exhibited normal morphology. (A) Images of female and male bladders from WT and SMNRKO mice. (B, C and D) SMNRKO mice (female, $\mathrm{n}=9$; male, $\mathrm{n}$ $=6$ ) had similar body weight, bladder weight, and bladder-to-body weight ratio compared with WT mice (female, $n=7$; male, $n=7$ ). (E, F, G and H) Representative Masson's trichrome staining images of bladder sections from female WT mice and SMNRKO mice. (I and J) Bladder sections from female WT and SMNRKO mice were subjected to immunofluorescence staining with anti- $\beta 1$ integrin antibody (green) and DAPI for nuclei (blue). (K, L, M and N) Representative Masson's trichrome staining images of bladder sections from male WT mice and SMNRKO mice. (O and $\mathrm{P}$ ) Bladder sections from male WT and SMNRKO mice were subjected to immunofluorescence staining with anti- $\beta 1$ integrin antibody and DAPI for nuclei, respectively. Data are shown as boxes and whiskers, the centerline is the median of the data set, the box represents $75 \%$ of the data, and bars indicate whiskers from minimum to maximum. Data were analyzed using the Student $t$ test. ${ }^{*} \mathrm{P}<0.05,{ }^{* *} \mathrm{P}<0.001$ compared with female or male WT mice.

Figure 2. SMNRKO mice exhibited a normal voiding pattern and urodynamic function. (A, B, C and D) Representative filters showed ultraviolet light-illuminated urine spots from female and male WT mice (female, $\mathrm{n}=32$ filters; male, $\mathrm{n}=26$ filters) and SMNRKO mice (female, $\mathrm{n}=44$ 
604 filters; male, $\mathrm{n}=24$ filters). (E, $\mathrm{F}$ and G) Summarized data of the numbers of primary voiding 605 spots (PVS: voiding spot area $\geq 80 \mathrm{~mm}^{2}$ ), area of PVS, and the total area of voiding spots per filter 606 indicate normal voiding patterns of SMNRKO mice. (H and I) Summarized frequency distribution 607 charts of spot size from female and male SMNRKO mice indicate similar voiding patterns of 608 SMNRKO mice with WT mice. (J and K) Representative CMG traces from female WT and 609 SMNRKO mice. (L, M, N, O and P) Summarized data of CMGs from female WT $(\mathrm{n}=5)$ and $610 \operatorname{SMNRKO}(\mathrm{n}=5)$ mice indicate a normal urodynamic function of SMNRKO mice. Data are shown 611 as box and whiskers, the centerline is the median of the data set, the box represents $75 \%$ of the 612 data, and bars indicate whiskers from minimum to maximum. Data were analyzed by the Student 613 t test. ${ }^{*} \mathrm{P}<0.05,{ }^{* *} \mathrm{P}<0.001$ compared with female or male WT mice.

614

615 Figure 3. SMNRKO mice had normal BSM contractility. (A, B, C and D) Representative traces of 616 BSM contraction from female and male WT mice (female, $\mathrm{n}=6$; male, $\mathrm{n}=8$ ) and $S M N R K O$ 617 (female, $\mathrm{n}=6$; male, $\mathrm{n}=7$ ) mice in response to $\mathrm{EFS}(1,2,5,10,20$, and $50 \mathrm{~Hz}$ ). (E and F) 618 Summarized data of EFS-stimulated contraction from SMNRKO mice showed similar contraction 619 force as in WT mice. (G, H and I) Summarized data of KCl-induced contraction, $\alpha, \beta$-meATP620 induced contraction, and carbachol-induced contraction from female and male SMNRKO mice 621 (female, $\mathrm{n}=8$; male, $\mathrm{n}=8$ ) exhibited normal BSM contractility with WT mice (female, $\mathrm{n}=6$; 622 male, $n=8$ ). Data are shown as box and whiskers, the centerline is the median of the data set, the 623 box represents $75 \%$ of the data, and bars indicate whiskers from minimum to maximum. Data were 624 analyzed by the Student t test. ${ }^{*} \mathrm{P}<0.05,{ }^{* *} \mathrm{P}<0.001$ compared with female or male WT mice.

626 Figure 4. Deficiency of NMDAR in BSM did not impact pathological bladder morphology. (A) 627 The images of bladders from female WT and SMNRKO mice pretreated with CYP or CYP and $628 \mathrm{MK} 801$. (B, C and D) SMNRKO mice pretreated with CYP $(\mathrm{n}=6)$ or CYP and MK801 $(\mathrm{n}=6)$ had 629 a similar abnormal body weight, bladder weight, and bladder-to-body weight ratio as WT mice 630 pretreated with CYP $(n=6)$ or CYP and MK801 $(n=6)$. MK801 reversed the increased bladder 631 weight and the ratio value, but not the decreased body weight induced by CYP in both WT and 632 SMNRKO mice. (E, F, G and H) Representative Masson's trichrome staining images of bladder 
633 cross-sections from a WT mouse pretreated with CYP. (I, J, K and L) Representative Masson's 634 trichrome staining images of bladder cross-sections from an SMNRKO mouse pretreated with 635 CYP. (M, N, O and P) Representative Masson's trichrome staining images of bladder cross636 sections from a WT mouse pretreated with CYP and MK801. (Q, R, S and T) Representative 637 Masson's trichrome staining images of bladder cross-sections from an SMNRKO mouse pretreated 638 with CYP and MK801. The urothelium (UR), lamina propria (LP), and bladder smooth muscle 639 (BSM) layers are indicated. The dashed line indicates the layers of UR, LP, or BSM. The black 640 triangles indicate the thin and disrupted urothelium. The black star indicates edema with enlarged 641 spaces in the LP. The black arrowheads in the muscle layer indicate enlarged spaces within muscle 642 bundles. Data are shown as box and whiskers, the centerline is the median of the data set, the box 643 represents $75 \%$ of the data, and bars indicate whiskers from minimum to maximum. Data were 644 analyzed by the Student $\mathrm{t}$ test. ${ }^{*} \mathrm{P}<0.05,{ }^{* *} \mathrm{P}<0.001$ compared with normal female WT mice and 645 the control WT mice. n.s: No significance $(>0.05)$.

647 Figure 5. Deficiency of NMDAR in BSM affected pathological bladder voiding pattern and 648 urodynamic function. (A, B, C and D) Representative filters showing UV light-illuminated urine 649 spots from female WT mice (CYP, $\mathrm{n}=20$ filters; CYP-MK801, $\mathrm{n}=24$ filters) and $S M N R K O$ 650 (CYP, $n=20$ filters; CYP-MK801, $\mathrm{n}=22$ ) mice pretreated with CYP or CYP and MK801. (E, F, $651 \mathrm{G}$ and H) Summarized data of numbers of PVS, area of PVS, total area of void spots per filter, and 652 frequency distribution of spot size indicate similar altered voiding patterns between WT and 653 SMNRKO mice pretreated with CYP or CYP and MK801. MK801 corrected the increased numbers 654 of PVS, decreased the area of PVS, and altered voiding patterns induced by CYP in both WT and 655 SMNRKO mice. (I, J, K and L) Representative CMG traces from female WT and SMNRKO mice 656 pretreated with CYP or CYP and MK801. (M, N, O, P and Q) Summarized data of CMGs from 657 female WT mice (CYP, $\mathrm{n}=4$; CYP-MK801, $\mathrm{n}=4)$ and SMNRKO mice (CYP, $\mathrm{n}=4$; CYP-MK801, $658 \mathrm{n}=4$ ) indicate similar altered urodynamic function between WT mice and SMNRKO mice 659 pretreated with CYP or CYP and MK801. MK801 reversed the decreased voiding interval, peak 660 pressure, compliance, and increased basal pressure induced by CYP in both mice. Data are shown 661 as box and whiskers, the centerline is the median of the data set, the box represents $75 \%$ of the 662 data, and bars indicate whiskers from minimum to maximum. Data were analyzed by the Student 
663 t test. ${ }^{*} \mathrm{P}<0.05,{ }^{* *} \mathrm{P}<0.001$ compared with normal female WT mice and control WT mice. n.s: 664 No significance $(>0.05)$.

665

666 Figure 6. Deficiency of NMDAR in BSM had no effects on pathological bladder BSM 667 contractility. (A, B, C and D) Representative traces of BSM contraction from female WT (CYP, $\mathrm{n}$ $668=9$; CYP-MK801, $\mathrm{n}=9)$ and SMNRKO (CYP, $\mathrm{n}=9$; CYP-MK801, $\mathrm{n}=9)$ mice pretreated with 669 CYP or CYP and MK801in response to $\operatorname{EFS~(1,~2,~5,~10,~20,~and~} 50 \mathrm{~Hz})$. (E, F, G and H) 670 Summarized data of EFS-induced contraction, KCl-induced contraction, carbachol-induced 671 contraction, and $\alpha, \beta$-meATP-induced contraction from SMNRKO mice pretreated with CYP or 672 CYP and MK801 showed similar contraction force with WT mice pretreated with CYP or CYP 673 and MK801. MK801 reversed reduced contraction force induced by CYP in both WT and 674 SMNRKO mice. Data are shown as box and whiskers, the centerline is the median of the data set, 675 the box represents $75 \%$ of the data, and bars indicate whiskers from minimum to maximum. Data 676 were analyzed using the Student $\mathrm{t}$ test. ${ }^{*} \mathrm{P}<0.05,{ }^{* *} \mathrm{P}<0.001$ compared with normal female WT 677 mice and the control WT mice. n.s: No significance $(>0.05)$.

678

679 Figure 7. Direct instillation of NMDAR agonists and antagonists into the CYP-induced cystitis 680 bladder did not impact urodynamic function. (A, B, C, D, E and F) Representative CMG traces 681 from female WT mice pretreated with CYP during the bladder infused with NMDAR agonists and 682 antagonists, including $100 \mu \mathrm{M}$ NMDA (n = 4), $100 \mu \mathrm{M}$ RS-tetrazol-5-yl glycine $(\mathrm{n}=4), 100 \mu \mathrm{M}$ 683 D-AP5 (n=4), $100 \mu \mathrm{M}$ CGS $19755(\mathrm{n}=4)$, and $100 \mu \mathrm{M}$ MK $801(\mathrm{n}=6) .(\mathrm{G}, \mathrm{H}, \mathrm{I}, \mathrm{J}$ and K) 684 Summarized data of CMGs indicate that NMDAR agonist or antagonist instillation did not impact 685 urodynamic function. Data are shown as box and whiskers, the centerline is the median of the data 686 set, the box represents $75 \%$ of the data, and bars indicate whiskers from minimum to maximum. 687 Data were analyzed using Student $t$ test. ${ }^{*} \mathrm{P}<0.05,{ }^{* *} \mathrm{P}<0.001$ compared with control. 


\section{Figure 1}

Bladders of SMNRKO mice exhibited normal morphology.

(A) Images of female and male bladders from WT and SMNRKO mice. (B, C and D) SMNRKO mice (female, $n=9$; male, $n=6$ ) had similar body weight, bladder weight, and bladder-tobody weight ratio compared with WT mice (female, $n=7$; male, $n=7)$. ( $E, F, G$ and $H$ ) Representative Masson's trichrome staining images of bladder sections from female WT mice and SMNRKO mice. (I and J) Bladder sections from female WT and SMNRKO mice were subjected to immunofluorescence staining with anti- $\beta 1$ integrin antibody (green) and DAPI for nuclei (blue). (K, L, M and N) Representative Masson's trichrome staining images of bladder sections from male WT mice and SMNRKO mice. ( $O$ and P) Bladder sections from male WT and SMNRKO mice were subjected to immunofluorescence staining with anti- $\beta 1$ integrin antibody and DAPI for nuclei, respectively. Data are shown as boxes and whiskers, the centerline is the median of the data set, the box represents $75 \%$ of the data, and bars indicate whiskers from minimum to maximum. Data were analyzed using the Student $t$ test. ${ }^{*} \mathrm{P}<0.05,{ }^{* *} \mathrm{P}<0.001$ compared with female or male WT mice. 

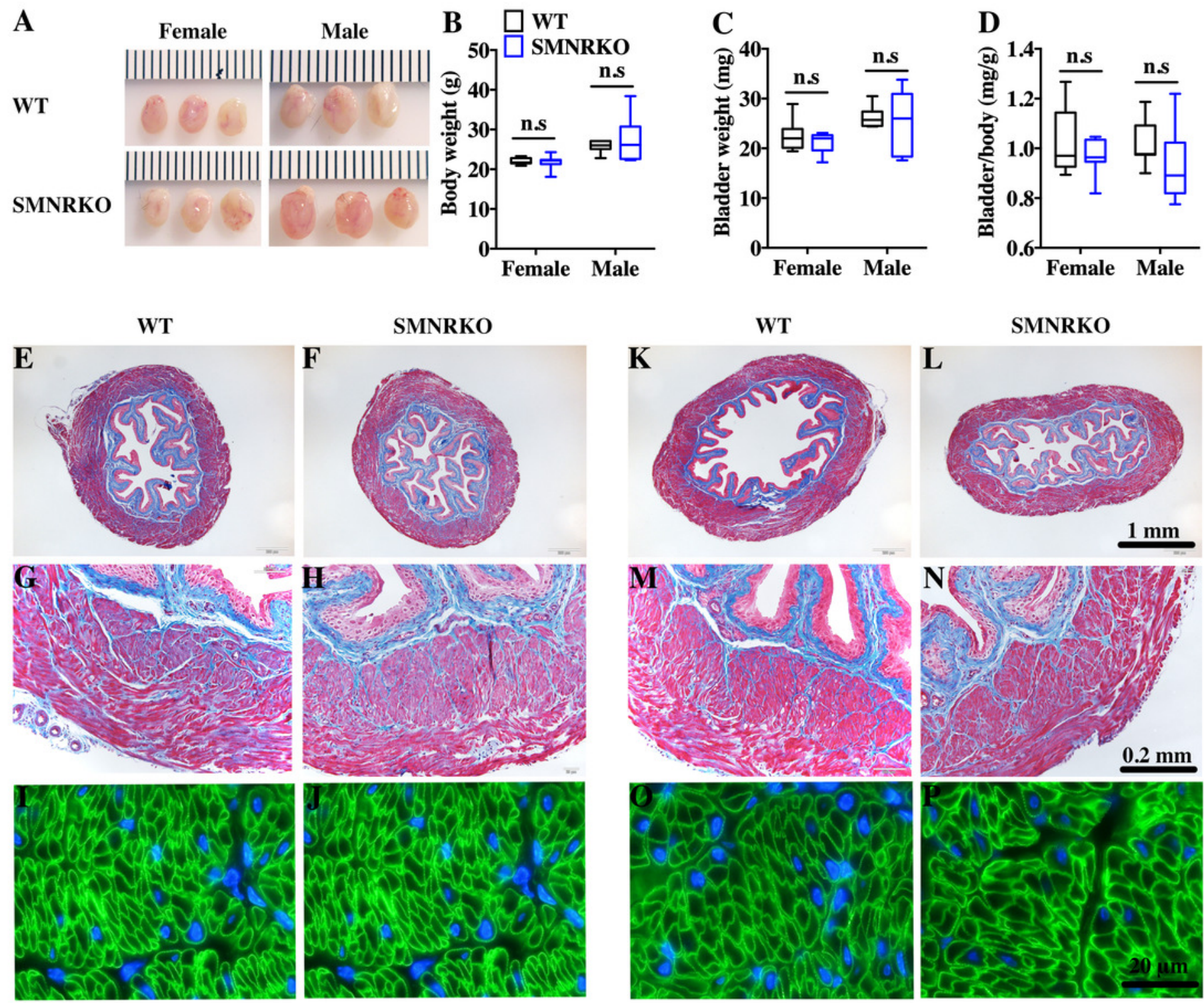

Female

Male 


\section{Figure 2}

SMNRKO mice exhibited a normal voiding pattern and urodynamic function.

(A, B, C and D) Representative filters showed ultraviolet light-illuminated urine spots from female and male WT mice (female, $\mathrm{n}=32$ filters; male, $\mathrm{n}=26$ filters) and SMNRKO mice (female, $n=44$ filters; male, $n=24$ filters). ( $E, F$ and G) Summarized data of the numbers of primary voiding spots (PVS: voiding spot area $\geq 80 \mathrm{~mm}^{2}$ ), area of PVS, and the total area of voiding spots per filter indicate normal voiding patterns of SMNRKO mice. ( $\mathrm{H}$ and I) Summarized frequency distribution charts of spot size from female and male SMNRKO mice indicate similar voiding patterns of SMNRKO mice with WT mice. (J and K) Representative CMG traces from female WT and SMNRKO mice. (L, M, N, O and P) Summarized data of CMGs from female WT $(n=5)$ and SMNRKO $(n=5)$ mice indicate a normal urodynamic function of SMNRKO mice. Data are shown as box and whiskers, the centerline is the median of the data set, the box represents $75 \%$ of the data, and bars indicate whiskers from minimum to maximum. Data were analyzed by the Student $t$ test. ${ }^{*} \mathrm{P}<0.05$, ${ }^{* *} \mathrm{P}<0.001$ compared with female or male WT mice. 

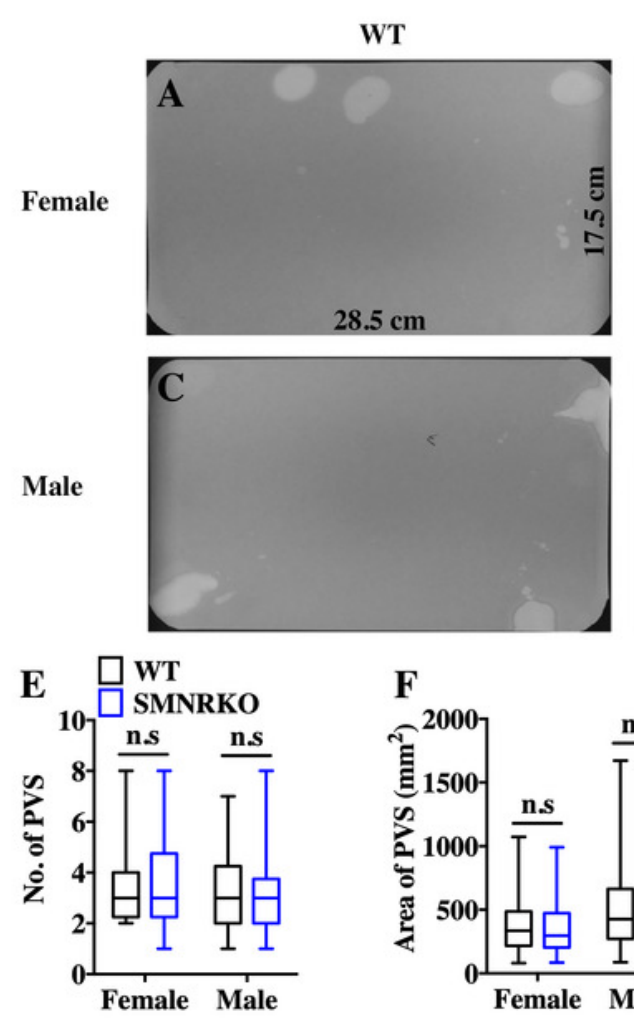

H

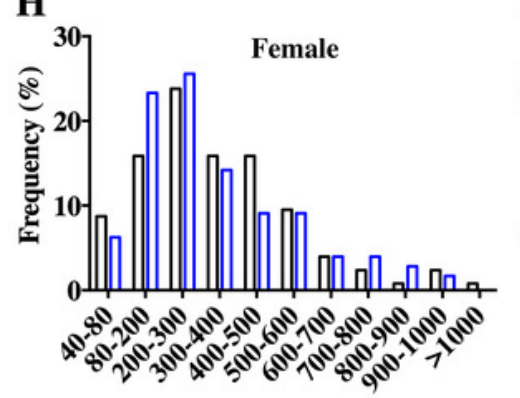

Size $\left(\mathrm{mm}^{2}\right)$

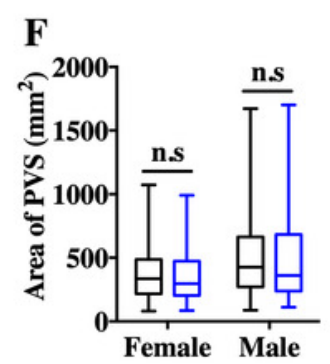

I

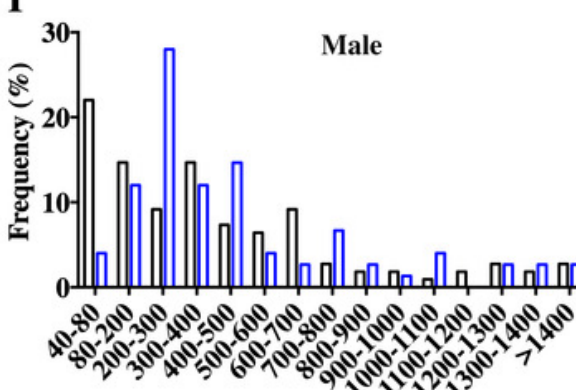

Size $\left(\mathrm{mm}^{2}\right)$

G

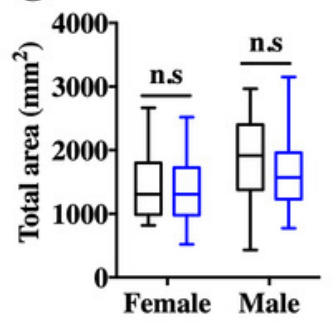

L
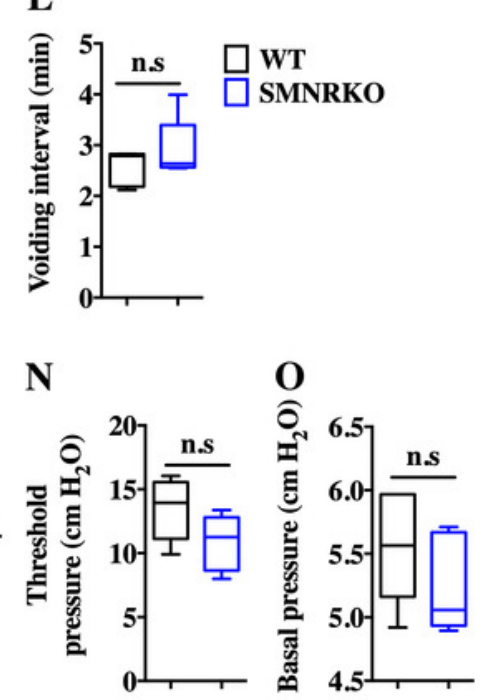

WT

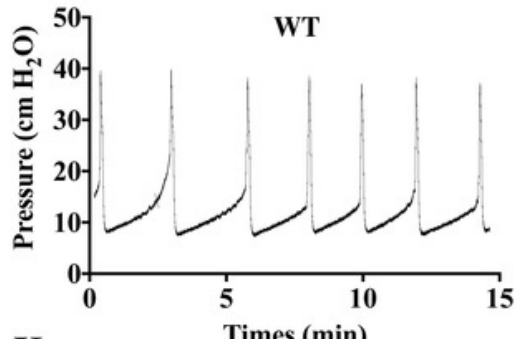

K
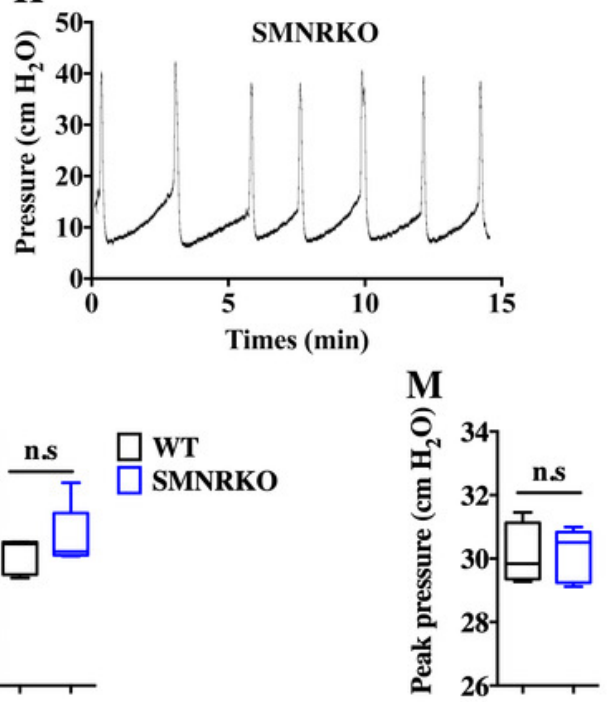

$\mathbf{P}$

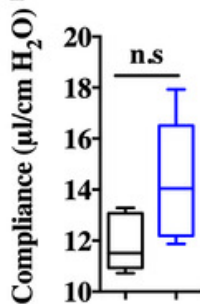




\section{Figure 3}

SMNRKO mice had normal BSM contractility.

( $A, B, C$ and D) Representative traces of BSM contraction from female and male WT mice (female, $\mathrm{n}=6$; male, $\mathrm{n}=8$ ) and SMNRKO (female, $\mathrm{n}=6$; male, $\mathrm{n}=7$ ) mice in response to EFS $(1,2,5,10,20$, and $50 \mathrm{~Hz})$. (E and F) Summarized data of EFS-stimulated contraction from SMNRKO mice showed similar contraction force as in WT mice. (G, $\mathrm{H}$ and I) Summarized data of KCl-induced contraction, $\alpha, \beta$-meATP-induced contraction, and carbachol-induced contraction from female and male SMNRKO mice (female, $\mathrm{n}=8$; male, $\mathrm{n}=8$ ) exhibited normal BSM contractility with WT mice (female, $n=6$; male, $n=8$ ). Data are shown as box and whiskers, the centerline is the median of the data set, the box represents $75 \%$ of the data, and bars indicate whiskers from minimum to maximum. Data were analyzed by the Student $\mathrm{t}$ test. ${ }^{*} \mathrm{P}<0.05,{ }^{* *} \mathrm{P}<0.001$ compared with female or male WT mice. 


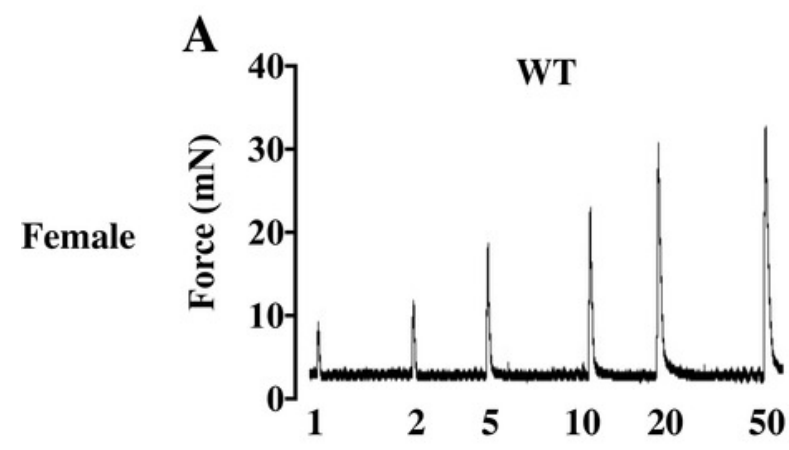

Frequnecy (Hz)
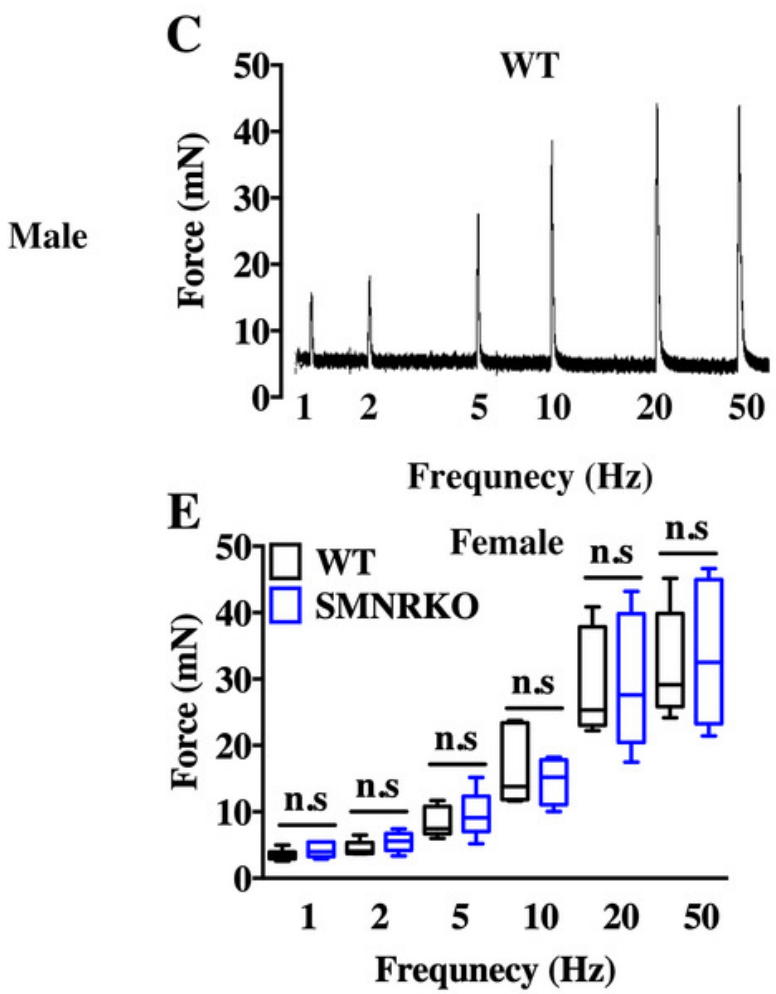
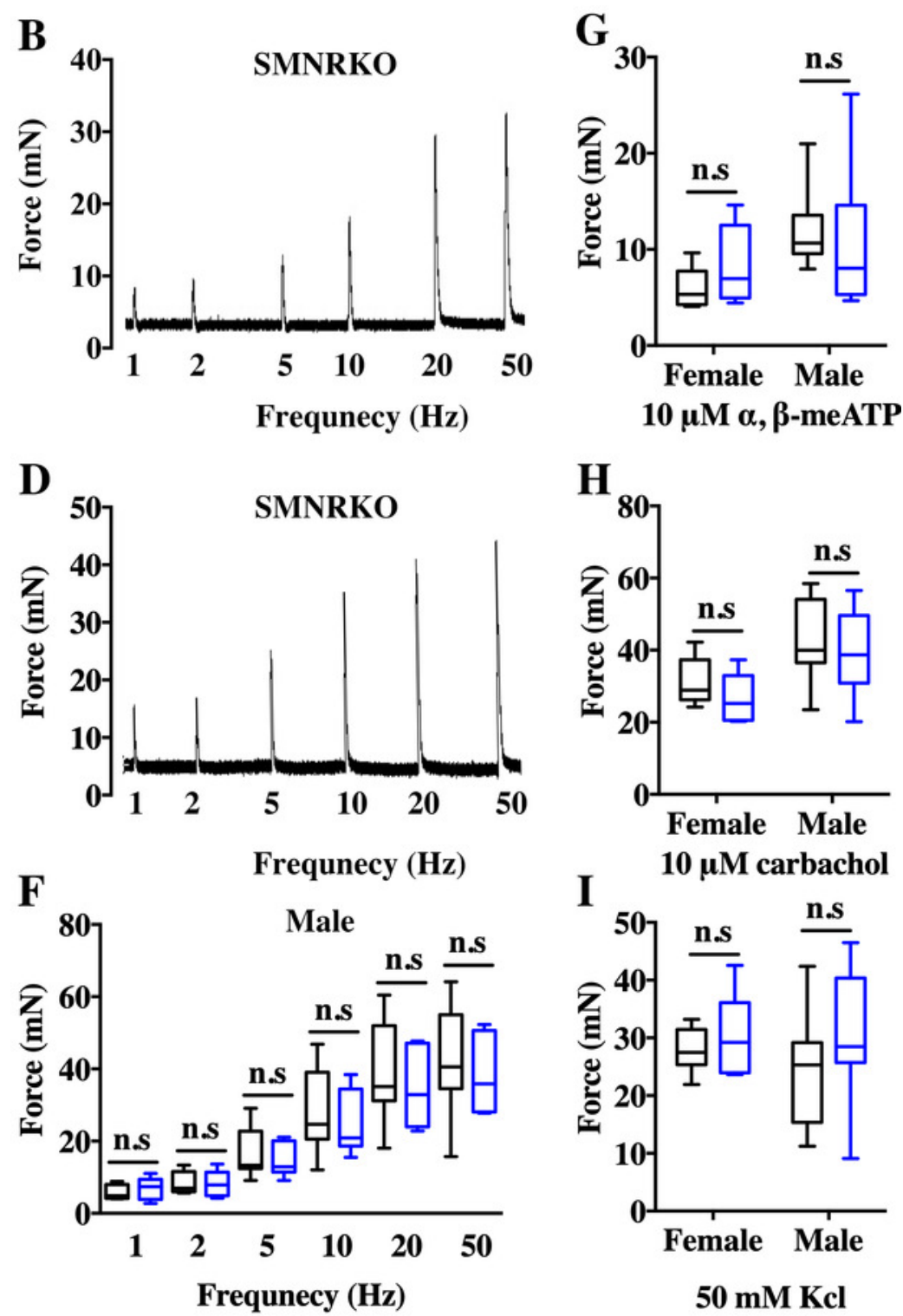
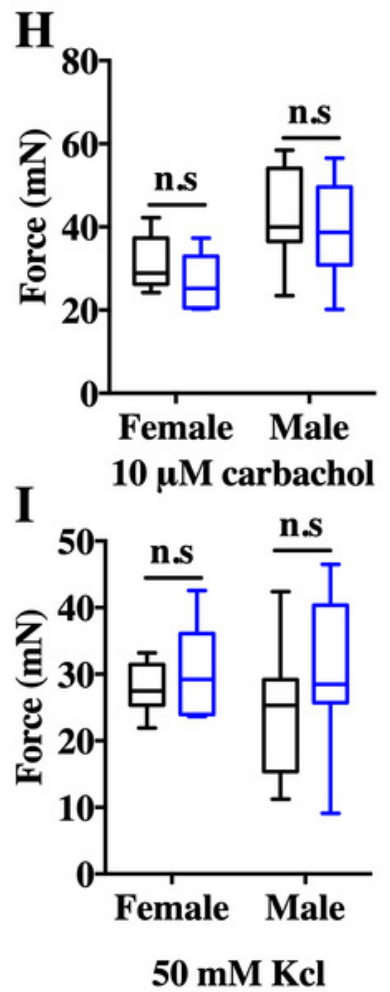


\section{Figure 4}

Deficiency of NMDAR in BSM did not impact pathological bladder morphology.

(A) The images of bladders from female WT and SMNRKO mice pretreated with CYP or CYP and MK801. (B, C and D) SMNRKO mice pretreated with CYP $(n=6)$ or CYP and MK801 $(n=6)$ had a similar abnormal body weight, bladder weight, and bladder-to-body weight ratio as WT mice pretreated with CYP $(n=6)$ or CYP and MK801 $(n=6)$. MK801 reversed the increased bladder weight and the ratio value, but not the decreased body weight induced by CYP in both WT and SMNRKO mice. (E, F, G and H) Representative Masson's trichrome staining images of bladder cross-sections from a WT mouse pretreated with CYP. (I, J, K and L) Representative Masson's trichrome staining images of bladder cross-sections from an SMNRKO mouse pretreated with CYP. (M, N, O and P) Representative Masson's trichrome staining images of bladder cross-sections from a WT mouse pretreated with CYP and MK801. ( $Q, R, S$ and $T$ ) Representative Masson's trichrome staining images of bladder cross-sections from an SMNRKO mouse pretreated with CYP and MK801. The urothelium (UR), lamina propria (LP), and bladder smooth muscle (BSM) layers are indicated. The dashed line indicates the layers of UR, LP, or BSM. The black triangles indicate the thin and disrupted urothelium. The black star indicates edema with enlarged spaces in the LP. The black arrowheads in the muscle layer indicate enlarged spaces within muscle bundles. Data are shown as box and whiskers, the centerline is the median of the data set, the box represents $75 \%$ of the data, and bars indicate whiskers from minimum to maximum. Data were analyzed by the Student $t$ test. ${ }^{*} \mathrm{P}<0.05,{ }^{* *} \mathrm{P}<0.001$ compared with normal female WT mice and the control WT mice. n.s: No significance $(>0.05)$. 


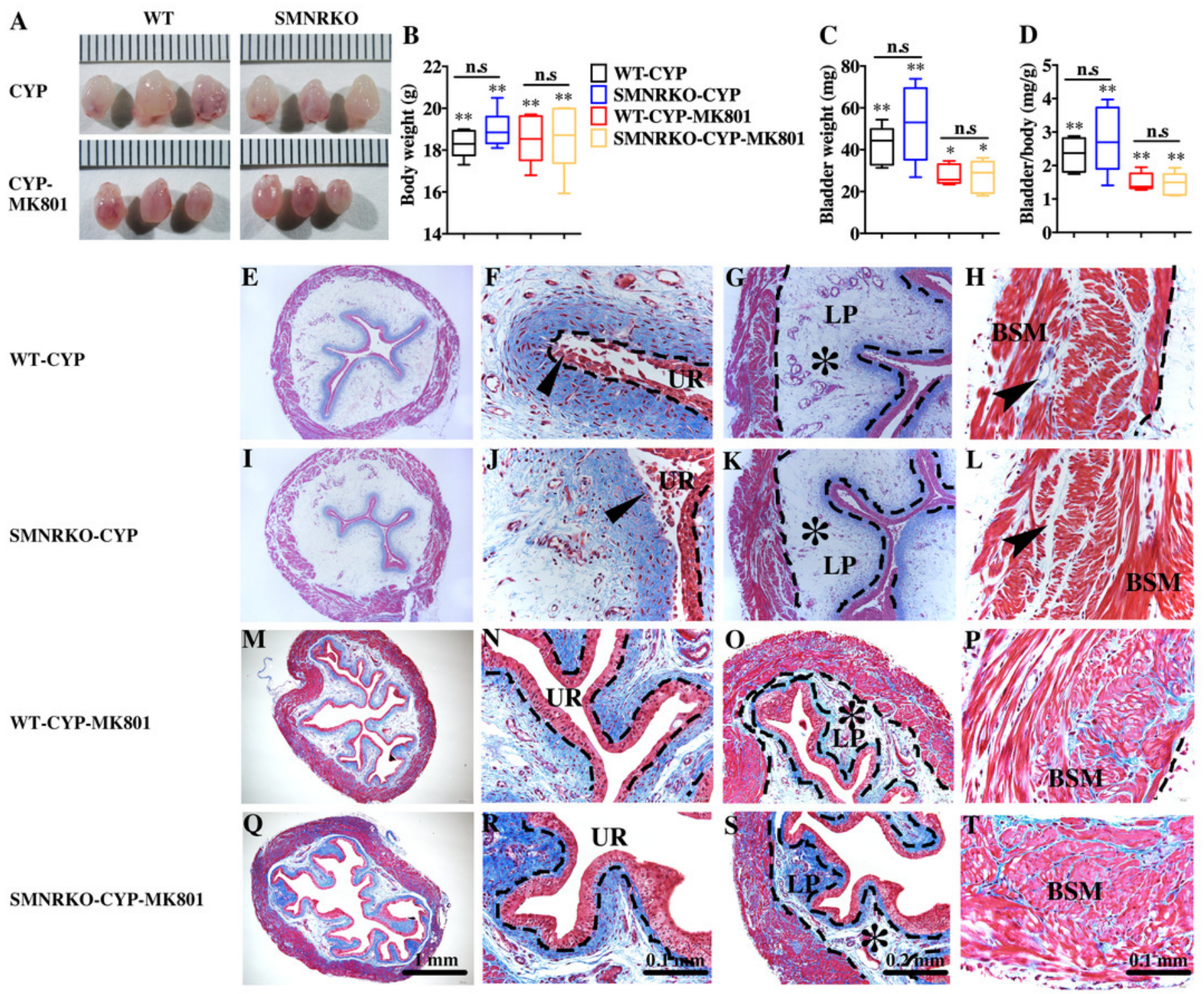




\section{Figure 5}

Deficiency of NMDAR in BSM affected pathological bladder voiding pattern and urodynamic function.

(A, B, C and D) Representative filters showing UV light-illuminated urine spots from female WT mice (CYP, $\mathrm{n}=20$ filters; CYP-MK801, $\mathrm{n}=24$ filters) and SMNRKO (CYP, $\mathrm{n}=20$ filters; CYP-MK801, $n=22$ ) mice pretreated with CYP or CYP and MK801. (E, F, G and H) Summarized data of numbers of PVS, area of PVS, total area of void spots per filter, and frequency distribution of spot size indicate similar altered voiding patterns between WT and SMNRKO mice pretreated with CYP or CYP and MK801. MK801 corrected the increased numbers of PVS, decreased the area of PVS, and altered voiding patterns induced by CYP in both WT and SMNRKO mice. (I, J, K and L) Representative CMG traces from female WT and SMNRKO mice pretreated with CYP or CYP and MK801. (M, N, O, P and Q) Summarized data of CMGs from female WT mice (CYP, $\mathrm{n}=4$; CYP-MK801, $\mathrm{n}=4$ ) and SMNRKO mice (CYP, $\mathrm{n}=4$; CYP-MK801, $n=4$ ) indicate similar altered urodynamic function between WT mice and SMNRKO mice pretreated with CYP or CYP and MK801. MK801 reversed the decreased voiding interval, peak pressure, compliance, and increased basal pressure induced by CYP in both mice. Data are shown as box and whiskers, the centerline is the median of the data set, the box represents $75 \%$ of the data, and bars indicate whiskers from minimum to maximum.

Data were analyzed by the Student $\mathrm{t}$ test. ${ }^{*} \mathrm{P}<0.05,{ }^{* *} \mathrm{P}<0.001$ compared with normal female WT mice and control WT mice. n.s: No significance (>0.05). 

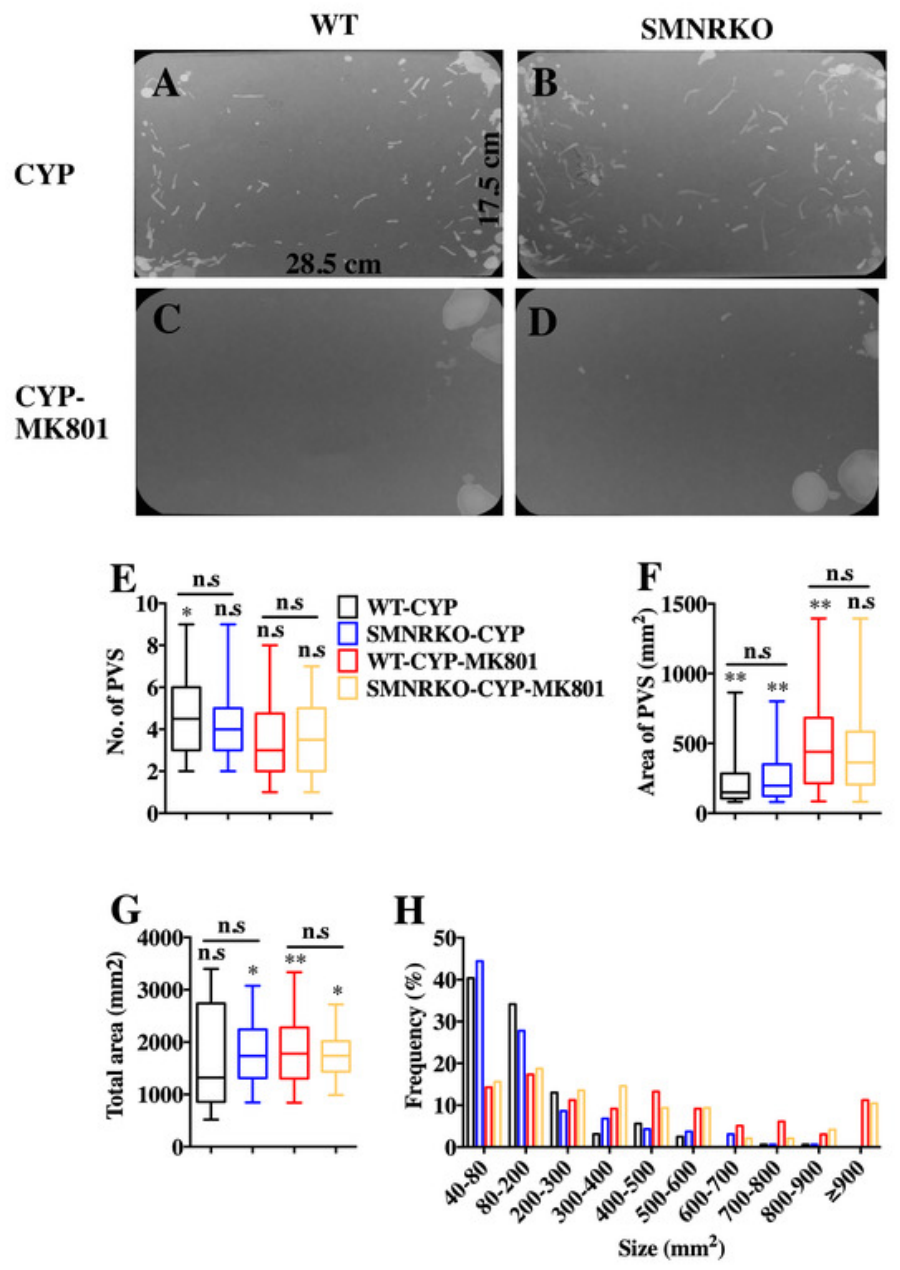
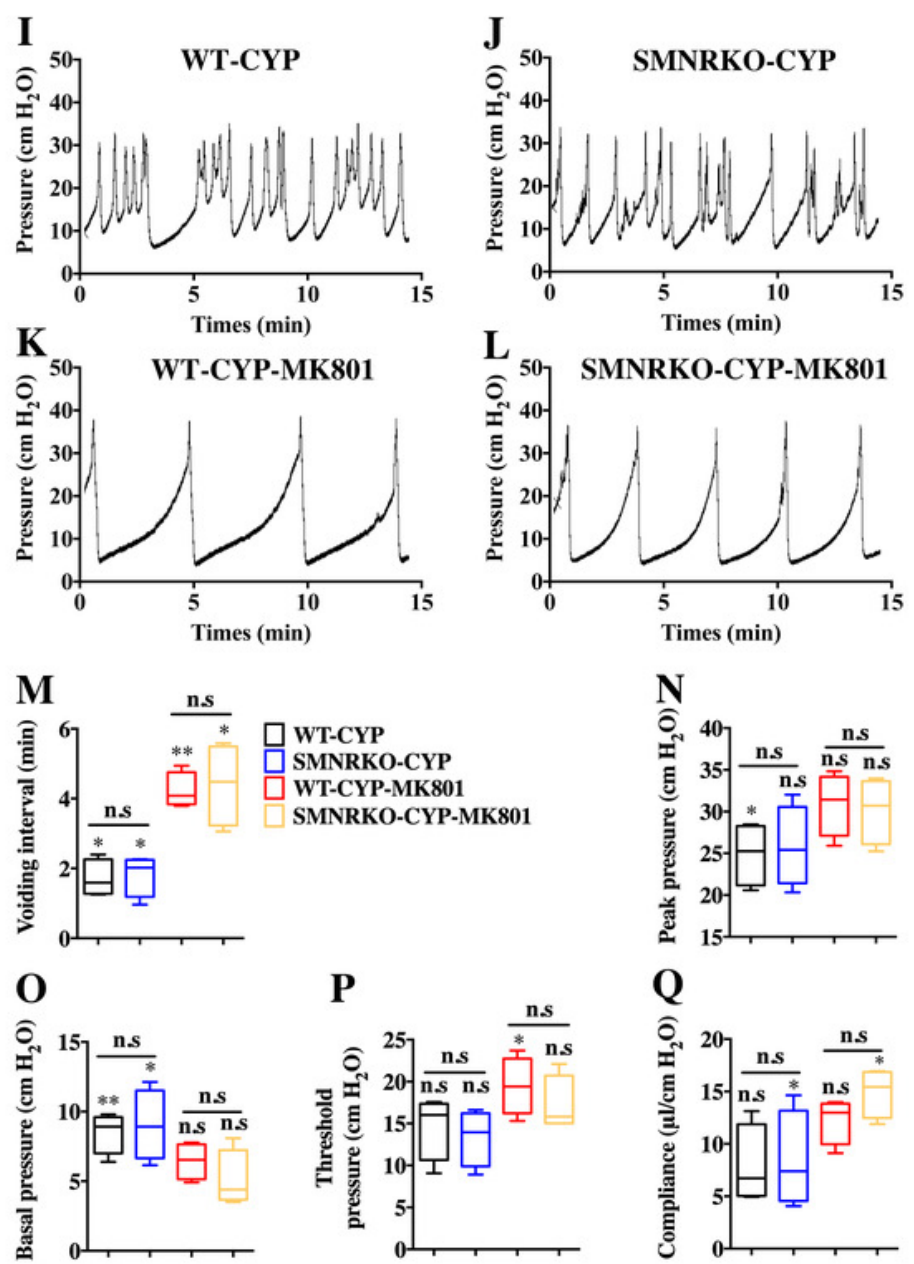

$\square$ WT-CYP

SMNRKO-CYP
WT-CYP-MK801

SMNRKO-CYP-MK801

Q
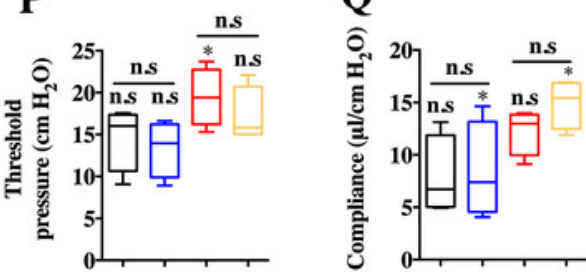


\section{Figure 6}

Deficiency of NMDAR in BSM had no effects on pathological bladder BSM contractility.

(A, B, C and D) Representative traces of BSM contraction from female WT (CYP, $n=9$; CYP-

MK801, $\mathrm{n}=9$ ) and SMNRKO (CYP, $\mathrm{n}=9$; CYP-MK801, $\mathrm{n}=9$ ) mice pretreated with CYP or CYP and MK801in response to $\operatorname{EFS}(1,2,5,10,20$, and $50 \mathrm{~Hz})$. (E, F, G and H) Summarized data of EFS-induced contraction, $\mathrm{KCl}$-induced contraction, carbachol-induced contraction, and a,bmeATP-induced contraction from SMNRKO mice pretreated with CYP or CYP and MK801 showed similar contraction force with WT mice pretreated with CYP or CYP and MK801. MK801 reversed reduced contraction force induced by CYP in both WT and SMNRKO mice. Data are shown as box and whiskers, the centerline is the median of the data set, the box represents $75 \%$ of the data, and bars indicate whiskers from minimum to maximum. Data were analyzed using the Student $\mathrm{t}$ test. ${ }^{*} \mathrm{P}<0.05,{ }^{* *} \mathrm{P}<0.001$ compared with normal female WT mice and the control WT mice. n.s: No significance $(>0.05)$. 

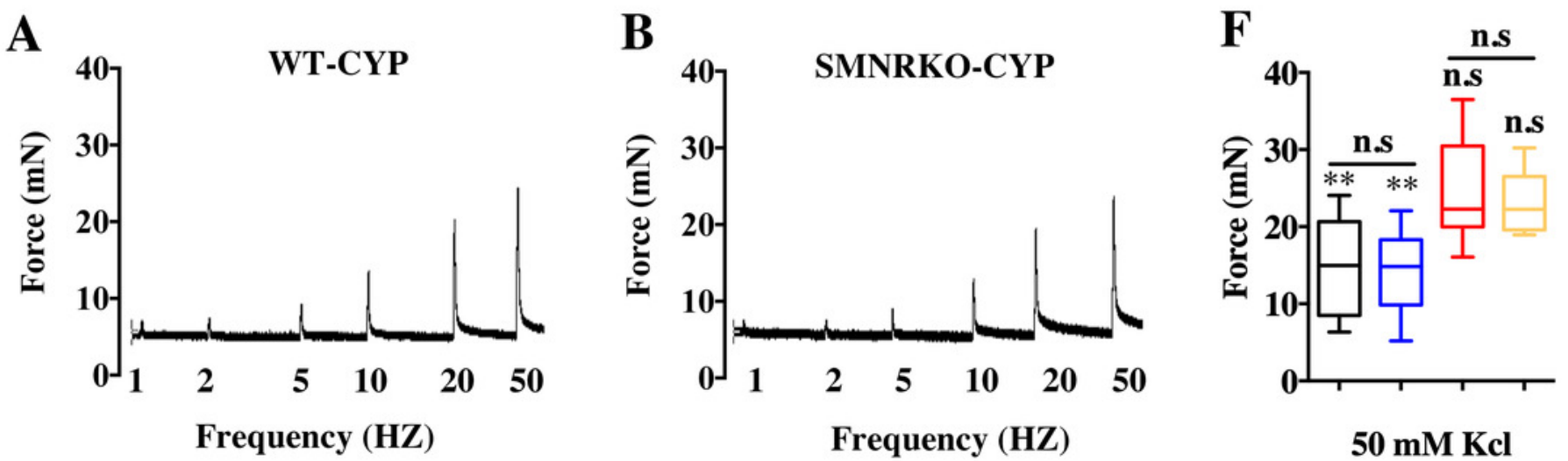

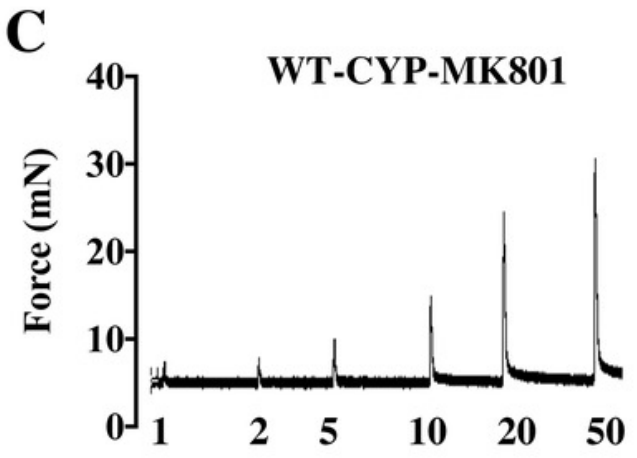

Frequency (HZ)
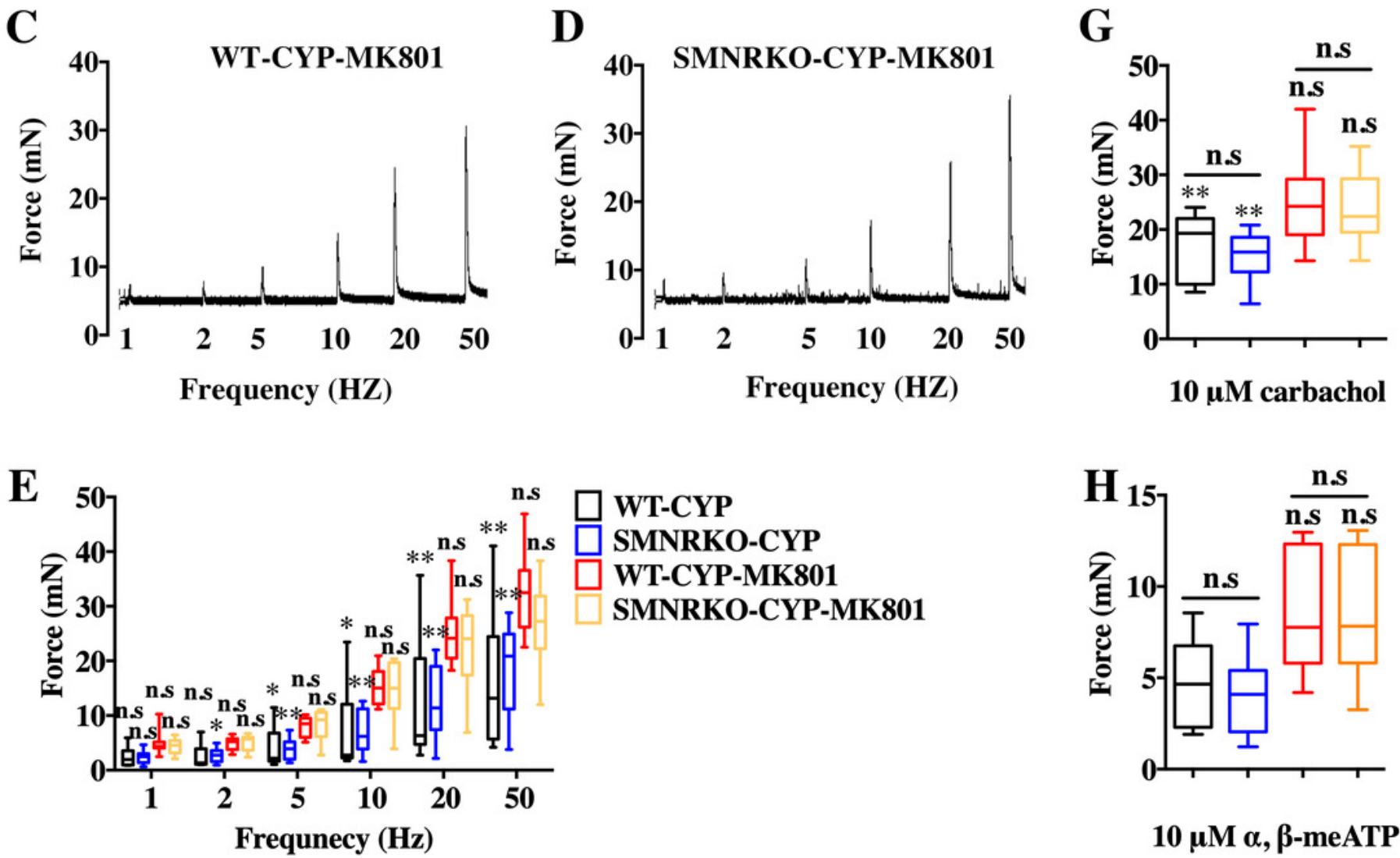

$10 \mu \mathrm{M} \alpha, \beta$-meATP 


\section{Figure 7}

Direct instillation of NMDAR agonists and antagonists into the CYP-induced cystitis bladder did not impact urodynamic function.

( $A, B, C, D, E$ and F) Representative CMG traces from female WT mice pretreated with CYP during the bladder infused with NMDAR agonists and antagonists, including $100 \mu$ M NMDA ( $\mathrm{n}$ = 4), $100 \mu$ M RS-tetrazol-5-yl glycine $(n=4), 100 \mu M$ D-AP5 ( $n=4), 100 \mu$ M CGS 19755 ( $n=$ 4), and $100 \mu M$ MK $801(n=6) .(G, H, I, J$ and K) Summarized data of CMGs indicate that NMDAR agonist or antagonist instillation did not impact urodynamic function. Data are shown as box and whiskers, the centerline is the median of the data set, the box represents $75 \%$ of the data, and bars indicate whiskers from minimum to maximum. Data were analyzed using Student $\mathrm{t}$ test. ${ }^{*} \mathrm{P}<0.05,{ }^{* *} \mathrm{P}<0.001$ compared with control.
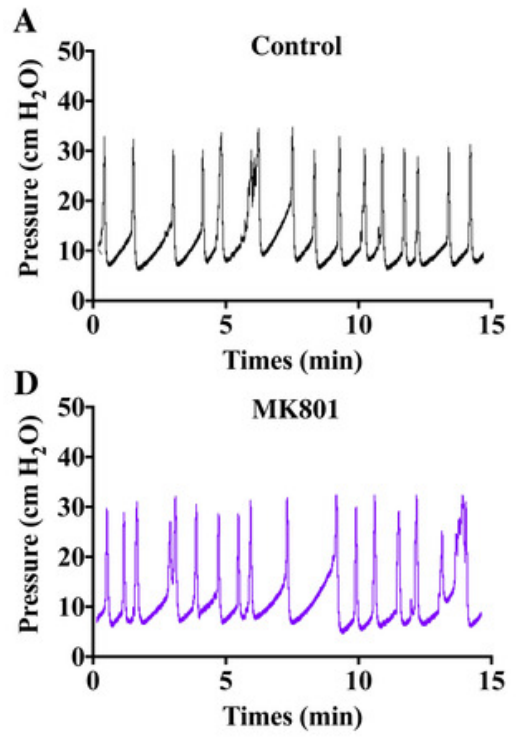

G

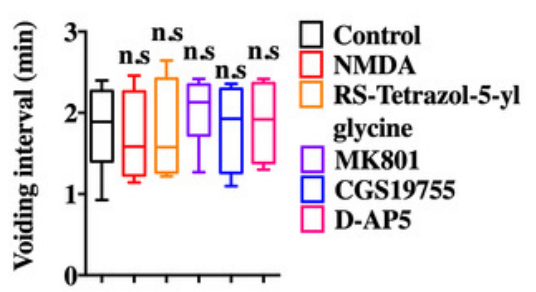

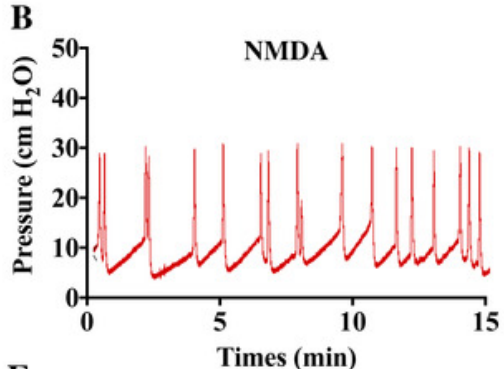
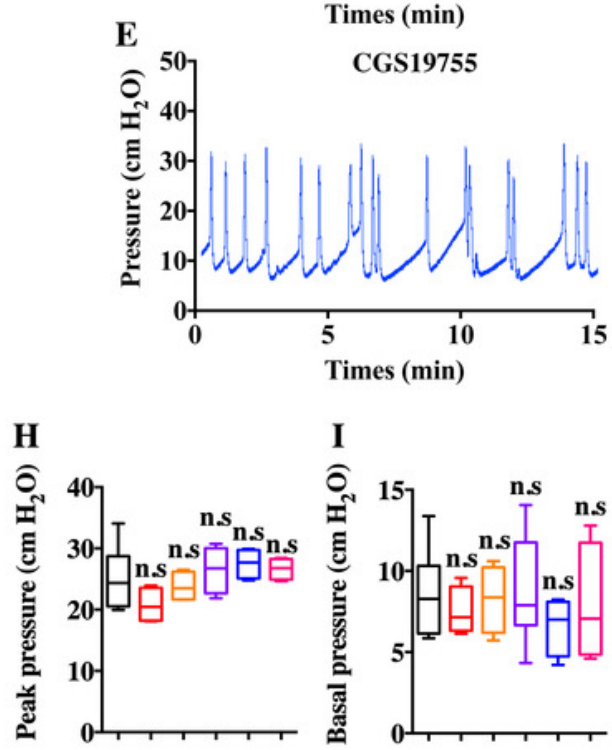

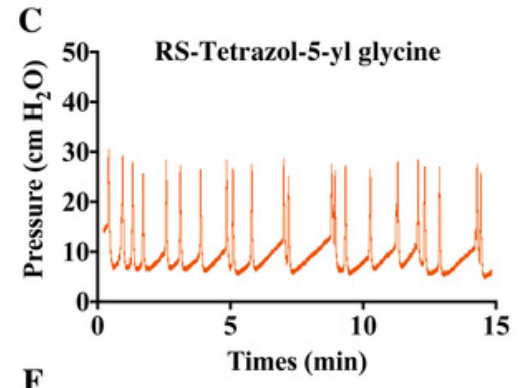

F

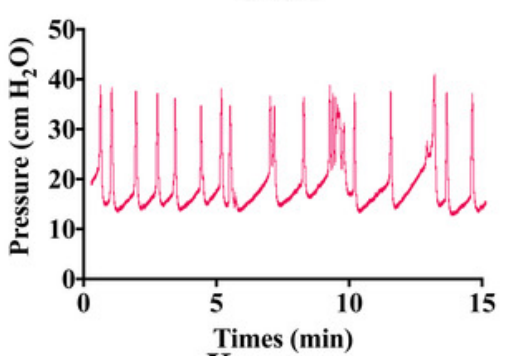

$\mathbf{J}$
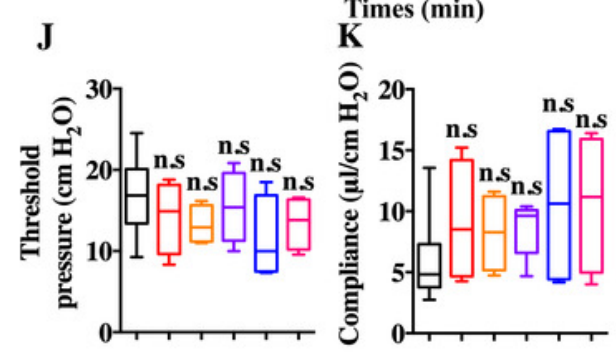